\title{
Neurophysiological and brain structural markers of cognitive frailty differ from Alzheimer's disease
}

Ece Kocagoncu ${ }^{1,2,4}$, David Nesbitt ${ }^{1,2}$, Tina Emery ${ }^{2}$, Laura Hughes ${ }^{1,2}$, Richard N. Henson ${ }^{2,3}$, James B. Rowe ${ }^{1,2,4}$ and Cam-CAN

1. Department of Clinical Neurosciences, University of Cambridge, Cambridge, CB2 OSZ, United Kingdom

2. MRC Cognition and Brain Sciences Unit, University of Cambridge, Cambridge, CB2 7EF, United Kingdom

3. Department of Psychiatry, University of Cambridge, Cambridge, CB2 OSZ, United Kingdom

4. Cambridge University Hospital NHS Foundation Trust, University of Cambridge, Cambridge, CB2 2QQ, United Kingdom

Correspondence to: Ece Kocagoncu

MRC Cognition and Brain Sciences Unit,

15 Chaucer Road

Cambridge, CB2 7EF

Email: ece.kocagoncu@cantab.net

Number of pages: 29

Number of figures: 4

Numbers of tables: 3

Number of figures in Extended data: 2

Number of tables in Extended data: 1

Number of words for abstract: 250

Number of words for introduction: 669

Number of words for discussion: 1500

\section{Conflict of interest}

The authors declare no competing financial interests.

\section{Acknowledgements}

The study was supported by the Medical Research Council [SUAG/004 RG91365, SUAG/046 RG101400; SUAG/051 RG101400], the Wellcome Trust [103838], the Dementias Platform UK [MR/L023784/1 \& MR/L023784/2], Alzheimer's Research UK [ARUK-PG2017B-19], a Holt Fellowship, and NIHR Cambridge Biomedical Research Centre (BRC-1215-20014). The views expressed are those of the authors and not necessarily those of the NIHR or the Department of Health and Social Care. For the purpose of open access, the author has applied a CC BY public copyright license to any Author Accepted Manuscript version arising from this submission. 


\begin{abstract}
With increasing life span and prevalence of dementia, it is important to understand the mechanisms of cognitive ageing. Here, we focus on a subgroup of the population we term "cognitively frail", defined by reduced cognitive function in the absence of subjective memory complaints, or a clinical diagnosis of dementia. Cognitive frailty is distinct from cognitive impairment caused by physical frailty. It has been proposed to be a precursor to Alzheimer's disease, but may alternatively represent one end of a non-pathological spectrum of cognitive aging. We test these hypotheses in humans of both sexes, by comparing the structural and neurophysiological properties of a community-based cohort of cognitive frail adults, to people presenting clinically with diagnoses of Alzheimer's disease or mild cognitive impairment, and community-based cognitively typical older adults. Cognitive performance of the cognitively frail was similar to those with mild cognitive impairment. We used a novel cross-modal pairedassociates task that presented images followed by sounds, to induce physiological responses of novelty and associative mismatch, recorded by EEG/MEG. Both controls and cognitively frail showed stronger mismatch responses and larger temporal grey matter volume, compared to people with mild cognitive impairment and Alzheimer's disease. Our results suggest that community-based cognitively frail represents a spectrum of normal ageing rather than incipient Alzheimer's disease, despite similar cognitive function. Lower lifelong cognitive reserve, hearing impairment and cardiovascular comorbidities might contribute to the aetiology of the cognitive frailty. Critically, community-based cohorts of older adults with low cognitive performance should not be interpreted as representing undiagnosed Alzheimer's disease.
\end{abstract}

\title{
Significance statement
}

The current study investigates the neural signatures of cognitive frailty in relation to healthy ageing and Alzheimer's disease. We focus on the cognitive aspect of frailty and show that despite performing similarly to the patients with mild cognitive impairment, a cohort of communitybased adults with poor cognitive performance do not show structural atrophy or neurophysiological signatures of Alzheimer's disease. Our results call for caution before assuming that cognitive frailty represents latent Alzheimer's disease. Instead the cognitive underperformance of cognitively frail adults could may a result of cumulative effects of multiple psychosocial risk factors over the lifespan, and medical comorbidities.

\section{Keywords}

Cognitive frailty; MEG; Aging; Alzheimer's disease; MRI; Cognitive reserve

\section{Abbreviations}

ACE-R: Addenbrooke's Cognitive Examination - Revised; Cam-CAN: Cambridge Centre for Ageing and Neuroscience; DA: Associative deviant; dB: Decibel; DN: Novelty deviant; EEG:

Electroencephalogram; ENT: Entorhinal cortex; GMV: Grey matter volume; HIP: Hippocampus; LHG: Left Heschl's gyrus; LIFG: Left inferior frontal gyrus; LSTG: Left superior temporal gyrus; MCl: Mild cognitive impairment; MEG: Magnetoencephalogram; MMSE: Mini Mental State Examination; MNI: Montreal Neurological Institute; MRI: Magnetic Resonance Imaging; PAL: Paired associated learning task; RHG: Right Heschl's gyrus; RIFG: Right inferior frontal gyrus; RSTG: Right superior temporal gyrus; RMS: Root-mean-square; ROI: Region of interest; STD: Standard; TIV: Total intracranial volume. 


\section{Author's contributions}

JBR, RNH, DN designed the study. LH and RNH designed the paradigm. DN, TE and EK collected the data. RNH preprocessed the structural MRI data. EK pre-processed the MEG data and performed all of the analyses. EK wrote the manuscript, and all authors contributed to the final version. 


\section{Introduction}

With longer life span and an older population, there is a pressing need to understand the mechanisms that determine cognitive ageing, and its relationship to dementias. The cognitively frail, is a population of interest, defined by reduced cognitive function in the absence of subjective memory complaints, or a clinical diagnosis of dementia, mild cognitive impairment $(\mathrm{MCl})$ or other pre-existing neurological explanation. Here cognitive frailty does not refer only to the cognitive impairments of those with comorbid physical frailty (Kelaiditi et al., 2013). Cognitive frailty has been linked to a higher risk of dementia, and is often regarded as a precursor to Alzheimer's disease (Panza et al., 2006; Buchman et al., 2007; Kojima et al., 2016; Shimada et al., 2018). In the absence of physical frailty, cognitive impairment alone is associated with longitudinal decline in functional abilities, activities of daily living (Shimada et al., 2016), increased hospitalisation and all-cause mortality rate (Avila-Funes et al., 2012; Solfrizzi et al., 2012; Ge et al., 2020).

However, there is an alternative hypothesis: poor cognitive performance reflects adverse aspects of normal ageing, without latent Alzheimer's disease or other neurodegenerative process. Psychosocial, educational, medical factors may contribute to cognitive frailty in the absence of latent degenerative or vascular dementia pathologies. For example cognitively under-performing adults are four times more likely to come from disadvantaged socioeconomic backgrounds, and twice as likely to have lower educational qualifications (Rogers et al., 2017). They are more likely to be malnourished (Mulero et al., 2011; Talegawkar et al., 2012; Chye et al., 2018), sedentary lifestyle (Landi et al., 2010; Rogers et al., 2017), have more medical comorbidities such as cardiovascular disease (Patrick et al., 2002; Langlois et al., 2012; Fuhrmann et al., 2019), chronic inflammation (Walston et al., 2002; Weaver et al., 2002; Cappola et al., 2003) and hearing impairment (Valentijn et al., 2005; Panza et al., 2015).

Here we determine whether cognitively frail community dwelling older adults have structural and/or neurophysiological characteristics of normal ageing or early Alzheimer's disease. We quantify brain structure and function using magnetic resonance imaging (MRI) and electromagnetoencephalography (E/MEG) respectively. In Alzheimer's disease, amyloid plaques and neurofibrillary tangles form early in entorhinal cortex and hippocampi (Hardy and Selkoe, 2002; Braak et al., 2006), leading to disruptions in synaptic and neural function (LaFerla and Oddo, 2005; West and Bhugra, 2015) and atrophy. If the cognitively frail have prodromal or undiagnosed Alzheimer's disease pathology, one would expect similar structural and neurophysiological changes. To assess the neural systems of hippocampal-dependent associative memory, we designed the cross-modal oddball task. The trials consisted of repeated pairings of an abstract image with a sound. A mismatch response arose from pairs that included either a novel sound (i.e. novelty deviant), or a sound that was not novel, but had been previously associated with a different image (i.e. associative deviant). The novelty deviants are akin to typical mismatch negativity responses; proposed to be an index of auditory predictive coding, which is attenuated in Alzheimer's disease (Ruzzoli et al., 2016; Laptinskaya et al., 2018). The associative deviants are a more sensitive test of Alzheimer's disease, since hippocampal dysfunction would impair the ability to establish cross-modal associations (Gottfried and Dolan, 2003; Joassin et al., 2011), and attenuate the response to associative deviants. Indeed, previous studies report impaired performance on the paired-associates learning task (PAL) in $\mathrm{MCl}$ and preclinical Alzheimer's disease, which correlates with disease progression (Blackwell et al., 2004; Ahmed et al., 2008). Moreover, in fMRI PAL shows Alzheimer's-related increased hippocampal activity and connectivity between hippocampus and cortical areas, (de Rover et al., 2011; Harrison et al., 2016). We measured novelty and associative deviant responses in lateral 
frontotemporal areas, readily detected by E/MEG in auditory oddball paradigms and reduced in dementia (Pekkonen, 2000; Garrido et al., 2009; Phillips et al., 2016).

We proposed that if cognitively frailty represents part of the spectrum of normal ageing, rather than latent Alzheimer's disease, then the neurophysiological responses and structural features of cognitively frail adults would resemble cognitively healthy adults rather than the patients with $\mathrm{MCl}$ or Alzheimer's disease.

\section{Materials and Methods}

\section{Study design}

The Cam-CAN Frail Project is an extension of the large-scale cross-sectional population-based Cambridge Centre for Ageing and Neuroscience study (Cam-CAN (Shafto et al., 2014)), focused on cognitive frailty. It examines the sub-population of community-dwelling adults with cognitive frailty, identified from home screening visits by cognitive screening tests: below $25 / 30$ on the MMSE and/or below 88/100 on the ACER in the absence of a diagnosis or referral for a memory disorder.

The Cam-CAN Frail protocol comprised of three sessions. First, a visit to the participant's home to assess lifestyle, health and cognitive performance on an extensive neuropsychological test battery. The battery included the revised Addenbrooke's Cognitive Examination (ACER), Mini Mental State Examination (MMSE), Wechsler Adult Intelligence Scale logical memory test, Spot the Word test, simple choice reaction time, famous faces test, four-mountains task, virtual object location and orientation, Rey figure recall, and the trail making test. In the second session, participants underwent E/MEG scanning and completed the Cattell and digit symbol tests. During the E/MEG recording, participants completed the cross-modal oddball task. In the final session, participants had a functional and structural magnetic resonance imaging and completed the Hotel task. The study was approved by the East of England - Cambridge Central Research Ethics Committee (10/H0308/50).

\section{Participants}

Participants consisted of community-dwelling older healthy controls, and patients diagnosed with either $\mathrm{MCl}$ or Alzheimer's disease by secondary healthcare services (Table 1). The cognitively frail individuals were defined by underperformance on cognitive tests, without any subjective memory complaints or clinical diagnosis of dementia, $\mathrm{MCl}$ or other significant neurological and psychiatric illness. A control group of cognitively successful healthy older adults was recruited, from the participants who had been assessed at home as part of the Cam-CAN 3000 home assessment (Shafto et al., 2014), but who had not been recruited into the "Cam-CAN 700" or "Cam-CAN 280" re-assessments. These healthy cognitive controls scored $>25 / 30$ on MMSE or $>88 / 100$ on the ACER during the home interview. The Cam-CAN home visits acquired lifestyle and cardiovascular risk characteristics (alcohol and smoking, hypertension, history of stroke and heart attack).

In addition, patients were recruited from local specialist memory clinics who had $\mathrm{MCl}$ or probable Alzheimer's disease diagnosed according to Petersen and McKhann criteria respectively (McKhann et al., 2011; Petersen et al., 2014). Most MCI/AD patients had positive cerebrospinal fluid biomarker status for Alzheimer's disease pathology, or clinical follow up to confirm the diagnosis. Participants were recruited from either sex, and were older than 50 years and were fluent speakers in English, with mental capacity to consent. Participants did not have any 
significant psychiatric illness or established neurological condition (other than $\mathrm{MCl}$ or Alzheimer's disease in the patient groups).

\section{E/MEG and MRI acquisition}

We used E/MEG to quantify neurophysiological dysfunction, as used in studies of healthy successful aging (Vlahou et al., 2014; Tsvetanov et al., 2015; Coquelet et al., 2017; Price et al., 2017), and early signatures of $\mathrm{MCl}$ and Alzheimer's disease (Osipova et al., 2005; Stam et al., 2006; de Haan et al., 2012; Maestú et al., 2015; Hughes et al., 2019; Kocagoncu et al., 2020). E/MEG data were acquired using the Elekta Vector View system with 204 planar gradiometers and 102 magnetometers. Simultaneous EEG data were acquired using a 70-channel Easycap. Participants' horizontal and vertical eye movements, and the cardiac activity were recorded using bipolar electro-oculogram and electro-cardiogram electrodes. Five head position indicator coils were placed on the EEG cap, to track the head position every 200 ms. For coregistration of the participant's T1-weighted MRI scan to the MEG sensors, three fiducial points (nasion, left and right pre-auricular) and a minimum of 100 head shape points were digitized using Polhemus digitization.

Participants were seated in a magnetically shielded room (IMEDCO) and positioned under the MEG scanner. Auditory stimuli were delivered binaurally through MEG-compatible ER3A insert earphones (Etymotic Research). The delay in sound delivery due to the length of earphone tubes and sound card was $26 \pm 2$ ms on average. Visual stimuli were presented on the screen positioned $1.22 \mathrm{~m}$ in front of the participant's visual field. Simultaneous E/MEG was recorded continuously at $1000 \mathrm{~Hz}$ with a high-pass filter of $0.03 \mathrm{~Hz}$. Before the E/MEG recording, participants performed an automated hearing test in the MEG scanner, to make sure that the earphones were working properly. They were presented pure tones at the frequency of $1000 \mathrm{~Hz}$ to either ear with varying loudness. Participants were instructed to press the button when they heard the tone. The mean hearing levels of each group is given in Table 1, where the normal range is expected to fall within $45-75 \mathrm{~dB}$.

T1-weighted structural images were acquired on a Siemens 3T Magnetom Prisma MRI Scanner using a MPRAGE sequence (repetition time $=2250 \mathrm{~ms}$, echo time $=2.99 \mathrm{~ms}$; inversion time $=$ $900 \mathrm{~ms}$; flip angle $=9$ degrees; field of view $=256 \mathrm{~mm} \times 240 \mathrm{~mm} \times 192 \mathrm{~mm}$; voxel size $=1 \mathrm{~mm}$ isotropic; GRAPPA acceleration factor $=2$; acquisition time $=4$ minutes 32 seconds). Four participants did not tolerate MRI due to claustrophobia.

\section{Stimuli}

The stimuli consisted of abstract images and pure tones. There were four images with distinct patterns. The tones had the following frequencies: $503 \mathrm{~Hz}, 719 \mathrm{~Hz}, 1021 \mathrm{~Hz}$ and $1451 \mathrm{~Hz}$. Harmonic tones were avoided by choosing frequencies of prime numbers and varying them by at least 3 semi-tones. There were four types of trials. 1) Standard (STD) trials were image-tone pairs that participants trained on prior to the task. Standard pairs were the trials presented most frequently. 2) Associative deviant (DA) trials presented the same images of the standard pairs but by shuffling the sounds. The DA trials were expected to capture the binding effect arising from a mismatch in association. 3) Novelty deviant (DN) trials presented the standard images with rare deviant tones. The frequencies used for the novel deviants were $599 \mathrm{~Hz}, 857 \mathrm{~Hz}, 1017 \mathrm{~Hz}, 1733$ $\mathrm{Hz}$. The DN trials were expected to capture the novelty effect, and were essentially the deviants used in conventional mismatch paradigms. The deviant trials were expected to induce a mismatch response with respect to the response to standard trials. 4) Target trials: The standard 
pairs, where the image was bound by a red circle. Target trials were included to make sure participants were attending to the stimuli. There were in total of 1000 standard trials and associative deviant, novel deviant and target trials were presented 48 times each. Therefore, the associative deviant, novel deviant and the targets were each encountered $4 \%$ of the time each, whereas standards $88 \%$ of the time.

\section{Paradigm}

The cross-modal oddball paradigm depends on both change detection and associative binding. This has two advantages. First, as MEG recording has lower signal to noise ratio in the subcortical areas and deeper sources compared to signal coming from superficial cortices (Goldenholz et al., 2009), the task was specifically designed to capture the indirect response in the superior temporal gyri and inferior frontal gyri, that are dependent on hippocampal associative learning. Secondly, the integrity of the auditory and frontal cortex is preserved until late stages of Alzheimer's disease, allowing us to control for atrophy of the cortical generators of the mismatch response. The task was easy to perform both by all participant groups, require minimal training (reducing potential confounds such as education and cognitive strategies on performance).

Images were presented centrally on a grey screen bounded by a black circle for $800 \mathrm{~ms}$. Then, $300 \mathrm{~ms}$ after image onset, the tone was played for $500 \mathrm{~ms}$ (Figure 1A). The $300 \mathrm{~ms}$ lag was introduced to allow participants to form predictions about the upcoming auditory stimuli. In between trials, a black fixation square was presented for a jittered period of 300-500 ms, resulting in a stimulus onset asynchrony between 1000-1200 ms. E-Prime 2 (Psychology Software Tools) was used to present the stimuli and send triggers to the scanner.

In the training phase, participants were presented in total four images and four tones (i.e. standard pairs), 25 times each, and were instructed to try to remember the pairings between the images and the tones. After the training, participants performed a short test where they listened to the four tones twice in a randomised order. After each tone, they were shown four images (i.e. chance level of \%25) on the screen and were asked to select the image that was paired with that tone. Irrespective of the participant's performance, training was repeated only once. Following the training participants moved on to the main task. Trials were presented in a different randomised order for each participant across four 5-minute long blocks. Participants were instructed to pay attention to the images and press the button with their right index finger when the image was bound by a red circle.

\section{E/MEG pre-processing and source localization}

The raw E/MEG data were pre-processed using MaxFilter 2.2.12 (Elekta Oy). Maxfiltering included detection and interpolation of bad sensors, signal space separation to remove external noise from the data and head movement correction. Cardiac and blink artefacts were detected and removed using an independent component analysis with 800 maximum steps and 64 principal components via the EEGLAB toolbox (Delorme and Makeig, 2004). The independent component time series were correlated with EOG and ECG time series and spatial templates. The components that revealed higher than $z=3$ in the temporal and $z=2$ in the spatial dimension were removed and the remaining time series of the independent components were reconstructed. On average 2.38 blink components $(S D=0.58)$ and 1.30 cardiac components $(S D=0.49)$ were removed.

Data were further processed in SPM12 (www.fil.ion.ucl.ac.uk/spm). Data were bandpass filtered between $0-40 \mathrm{~Hz}$ using a fifth-order Butterworth filter. The continuous data were epoched 
between -100 to 500 ms from the sound onset. OSL's artefact rejection algorithm (github.com/OHBA-analysis/osl-core) was used to remove remaining artefacts (e.g. motor). Bad channels and trials marked by the algorithm were removed. On average 53.04 ( $4.6 \%$; SD = 35.88) trials and 10.76 channels $(2.8 \% ; S D=5.85)$ were removed per participant. Trials were averaged within condition, using robust averaging. Low-pass filter was re-applied to correct for the highfrequency noise introduced by robust averaging.

The E/MEG data were source localised using all sensor data: magnetometers, gradiometers and EEG (Henson et al., 2009). The source space was modelled with a medium sized cortical mesh consisting of 8196 vertices via inverse normalization of SPM's canonical meshes. Sensor positions were coregistered to the native T1-weighted MPRAGE scans using the fiducial and head shape points after removing digitisation points around the nose. SPM's canonical template brain was used for participants who did not tolerate the MRI scan. Single shell and Boundary Element models were used for forward modelling of MEG and EEG data respectively. Evoked signal was estimated over the trials using the $\mathrm{COH}$ solution in SPM which imposes spatial smoothness on the prior covariance matrix. All inversion accuracies were above $80 \%$, as measured by the proportion of variance explained in the sensor data $(M=93.62 ; S D=3.63)$.

Although neurophysiological responses in the hippocampus are difficult to detect with E/MEG, owing to its depth, a strong mismatch response can be recorded from temporal cortex, where sensory predictions are assumed to be established from hippocampal-dependent cross-modal associative learning. We therefore focus on the mismatch response in the lateral temporal auditory and frontal cortex, as activated in conventional auditory oddball paradigms (Pekkonen, 2000; Garrido et al., 2009; Hughes and Rowe, 2013; Phillips et al., 2016; Hughes et al., 2018). The source localised data were extracted from 6 areas taken from the Automated Anatomical Labelling atlas: Heschl's gyri, superior temporal gyri and inferior frontal gyri bilaterally (Figure 2C). The ROI masks were resliced to $1 \mathrm{~mm}$ isotropic thickness to allow maximum data extraction. For each participant and condition, the data were extracted from the peak within all the vertices that constitute each ROI. This is to maximise the signal to noise ratio in the data, and to account for individual variability in source activity. We had two contrasts of interest in the analyses: the STDDN contrast captures the novelty mismatch effect, while the STD-DA contrast captures the associative mismatch effect.

\section{MRI pre-processing and grey matter analysis}

The T1 image was rigid-body co-registered to a Montreal Neurological Institute (MNI) template and then corrected for image inhomogeneity and segmented into 6 tissue classes (grey matter, white matter, cerebrospinal fluid, bone, soft tissue, and residual noise) using SPM's unified segmentation algorithm (Ashburner and Friston, 2005). The native space grey and white matter images for all participants were then submitted to diffeomorphic registration (DARTEL)

(Ashburner, 2007) to create group template images. The group template was then normalised to the MNI template via an affine transformation and the combined normalisation parameters (native to group template and group template to MNI template) were applied to each individual participant's grey matter image, including modulation in order to preserve local volume. Region of Interest (ROI) from the Harvard-Oxford atlas were then used to extract mean regional GMV from the bilateral hippocampal and entorhinal ROIs for each participant. The GMVs were compared across groups using ANCOVAs where age and total intracranial volume (TIV) were set as covariates.

To calculate local grey matter atrophy at the whole brain level we used voxel-based morphometry (VBM). Grey matter segments were thresholded with an absolute masking level of 
0.1 , and were smoothed with a Gaussian kernel at $8 \mathrm{~mm}$ full width half maximum. Grey matter volumes were compared across groups in pairwise t-contrasts in general linear models accounting for differences in age and total intracranial volume. The cluster level $p$-values were corrected for multiple comparisons using the family-wise error after a cluster defining threshold of $p<0.05$.

\section{RMS and statistical analyses}

To investigate differences in time series, the root-mean-square of the time series at each ROI and trial were smoothed using a moving average at every 50 time points, to remove jumps. The RMS at each time point was then modelled using general linear models (GLM) accounting for differences in age and hearing levels, and tested for within group task effects by using tcontrasts. The contrasts compared the signal intensity between the DA-STD and DN-STD. The tests comparing the deviant effects were performed firstly within each participant group, to reveal task-specific effects. Secondly, these differences were tested across groups to test for interaction effects between conditions and groups. The observed clustermasses in the GLMs were corrected for multiple comparisons using permutation cluster statistics, by bootstrapping the design matrix using 1000 permutations at $p=0.05$. The mean of the time series within each contrast were calculated for each participant within the 200-500 ms time window after removing outliers. This time window was selected because task effects were strongest after the N100. The linear relationship between these metrics and predictor variables were further tested through general linear models across the sample including age as a covariate, and after removing outliers. The predictors of interest were years of education, ACER total and memory subscale scores, and hippocampal and entorhinal grey matter volumes.

\section{Results}

\section{Sample characteristics}

Sample characteristics and scores on neuropsychological tests were compared across the groups using ANOVAs. Age $(F(3,87)=3.82 ; p=0.012)$ and years of education $(F(3,87)=11.34 ; p<0.001)$ differed between groups. Tukey's HSD tests showed that the cognitively frail group was older than controls $(p=0.006)$. The duration of formal education was longer in those in the control group than the cognitively frail ( $p<0.001)$, and Alzheimer's disease group $(p=0.032)$. The $\mathrm{MCl}$ group had had longer education than the cognitively frail $(p<0.001)$ and Alzheimer's disease $(p=$ $0.001)$ groups. Hearing levels were tested for group differences using both ANOVA and ANCOVA (to control for differences in age). There were no significant differences in hearing in the left ear. In the right ear there was a group difference $(F(3,86)=4.70 ; p=0.004)$ : the hearing of the cognitively frail was lower than control $(p=0.017)$ and $\mathrm{MCl}(p=0.005)$ groups. When adjusting for the differences in age $(F(3,85)=3.02 ; p=0.034)$, the hearing levels on the right were still lower in the cognitively frail group compared to the $\mathrm{MCl}$ group $(p=0.019)$. Chi-square tests compared the prevalence of lifestyle and cardiovascular risk factors between control and the cognitively frail groups. The prevalence of daily alcohol consumption was lower in the cognitively frail group (19\%) compared to controls $(43 \%)\left(X^{2}(1)=6.11 ; p=0.006\right)$. The prevalence of hypertension was higher in the cognitively frail group (52\%) compared to the controls (35\%) $\left(X^{2}(1)=2.74 ; p=0.048\right)$. There were no significant differences between groups in the prevalence of smoking, history of stroke or heart attack. 


\section{Cognitive results}

Cognitive scores were tested for group differences after controlling for differences in age (Table 1). The MMSE $(F(3,86)=17.64 ; p<0.001)$, ACER total score $(F(3,86)=55.41 ; p<0.001)$, ACER's subscales in memory $(F(3,86)=37.35 ; p<0.001)$, attention $(F(3,86)=9.05 ; p<0.001)$, fluency $(F(3,86)=13.87 ; p<0.001)$, language $(F(3,86)=7.90 ; p<0.001)$ and visuospatial skills $(F(3,86)=$ $11.15 ; p<0.001$ ) showed strong differences across the groups (Figure $1 \mathrm{~A})$. Results of the pairwise post-hoc comparisons are given in Figure 1B. The cognitively frail group performed similarly to the $\mathrm{MCl}$ group across all cognitive tests, except for the fluency subscale, where their scores were significantly lower than the $\mathrm{MCl}$ group $(p<0.001)$. All four groups performed above chance level on the training test. The scores were significantly different across groups $(F(3,86)=5.60 ; p=$ $0.001)$. Post-hoc comparisons showed that the controls performed significantly better than the $\mathrm{MCl}(p=0.015)$ and Alzheimer's disease $(p=0.006)$ groups. There were no significant differences between the training scores of the cognitively frail group and other groups.

\section{Grey matter atrophy}

Mean hippocampal GMV, entorhinal GMV, total GMV and total intracranial volume (TIV) were compared across the groups, corrected for age and TIV using ANCOVA. There were no significant differences between groups for TIV or total GMV. However, hippocampal $(F(3,86)=10.35 ; p<$ 0.001 ) and entorhinal $(F(3,86)=7.62 ; p<0.001)$ GMVs showed a main group effect (Figure $2 A-B$ ). The hippocampal GMV in the control group was significantly larger compared to the $\mathrm{MCl}(p<$ $0.001)$ and Alzheimer's disease groups $(p<0.001)$. Similarly, the entorhinal GMV of the control group was larger compared to the $\mathrm{MCl}(p=0.001)$ and Alzheimer's disease groups $(p=0.003)$. The hippocampal and entorhinal volumes of the cognitively frail group was similar to the control group.

Atrophy was tested at the voxel level, using VBM (Table 2). As expected, the control group had significantly higher GMV in bilateral temporal cortices and hippocampi compared to the $\mathrm{MCl}$ and $A D$. We found a similar pattern comparing cognitively frail group to $\mathrm{MCl}$ and Alzheimer's disease group, although cluster extents were smaller (Figure 2D-E). In order to confirm that the differences we observe in the neurophysiological responses could be due to local GM atrophy of the lateral frontotemporal areas, we tested the GMV of the lateral frontotemporal areas bilaterally (i.e. IFG, STG, HG), and report that the GMVs of the superficial areas do not differ across groups (Extended data, Figure 2-1).

\section{Cross-modal mismatch responses}

Figure 3A-D displays the gradiometer topoplots for each condition in $100 \mathrm{~ms}$ time windows across the groups. Following N100, topoplots show a strong burst of bilateral activity in frontal and temporal sensors that is sustained until the end of the epoch. Note that compared to the associative deviant and standard, novelty deviant induced a stronger and more widespread activity across the frontotemporal sensors. The gradiometer topoplots are given here for visualisation only; statistical comparisons were made in the a priori source space ROls.

We tested the time series of each deviant with respect to the standard, within the 6 ROIs (Table 3, Figure 3E). We found strongest effects for the novelty deviant in the bilateral IFG early in the epoch, following the onset of the sound. The effects seen in the cognitively frail group mirrored the controls. Further, novelty deviant effects were found across all the ROls in the control group. 
$\mathrm{MCl}$ and Alzheimer's disease groups showed no significant novelty effects in the IFG, and weaker clusters limited to STG and HG. Associative deviant effects were found in the IFG across all groups, and in overlapping time windows starting around $200 \mathrm{~ms}$ after the sound onset.

We tested for the interaction effects between the deviant responses (i.e. STD-DA, and STD-DN) and group. There was no significant interaction between the control and cognitively frail group or between $\mathrm{MCl}$ and Alzheimer's disease groups. However, the control group showed stronger associative and novelty deviant responses compared to both $\mathrm{MCl}$ and Alzheimer's disease groups. Similarly, cognitively frail group showed the same interaction effects against the $\mathrm{MCl}$ and Alzheimer's disease groups. As a confirmatory analysis, we also repeated these GLMs with logtransformed squared RMS time series to ensure that the normality assumption is met. These results can be found in the Extended data, Table 3-1.

\section{Clinical and structural correlates of the cross-modal mismatch}

To assess how the deviant responses relate to clinical severity, education and medial temporal lobe atrophy, the linear relationships between the E/MEG contrast means at 200-500 ms and each predictor variable were tested using general linear models (Figure 4A) whilst controlling for differences in age. This revealed strong relationships between the novelty deviant mean in the LHG and RHG with ACER total and ACER-memory subscale scores: the higher the scores on cognitive tests, the more negative (towards normal) the novelty deviant was. A strong negative relationship between the hippocampal and entorhinal volumes and the deviant response was observed for the left hemisphere ROIs, particularly the LHG. This suggests that medial temporal atrophy is associated with a reduced deviant response even though the MMN response arises from extra-hippocampal auditory cortex. This negative relationship was stronger for the novelty deviant compared to the associative deviant. Education showed moderate positive relationships with the associative deviant in the LSTG and RSTG, whereas it showed a negative relationship with the novelty deviant mean in RIFG.

We calculated the partial correlations amongst predictor variables correcting for differences in age. Education showed positive correlations with ACER total $(r=0.40 ; p<0.001)$ and ACER memory subscale scores $(r=0.35 ; p=0.001)$, but did not correlate with hippocampal and entorhinal volumes. ACER total score correlated with both hippocampal $(r=0.53 ; p<0.001)$ and entorhinal GMV $(r=0.40 ; p<0.001)$. Similarly, ACER memory subscale score positively correlated with hippocampal $(r=0.55 ; p<0.001)$ and entorhinal GMV $(r=0.41 ; p<0.001)$.

\section{Discussion}

The principal result of this study is that community-dwelling cognitively frail individuals do not resemble people with $\mathrm{MCl}$ or Alzheimer's disease, in terms of their structural or neurophysiological profile, despite similar levels of underperformance on cognitive screening tests. The poor cognitive performance of the cognitively frail participants should not simply be interpreted arising from latent Alzheimer pathology or undiagnosed amnestic $\mathrm{MCl}$. Population screening using standard cognitive tests (e.g. MMSE, or ACER) is therefore unlikely to selectively identify those with latent Alzheimer's disease pathology without additional biomarker evidence of pathology. There are other associations of cognitive impairment, including lower educational level, hearing impairment, and cardiovascular risk factors. Both structural and neurophysiological features of the cognitively frail group were similar to controls. Structural analyses revealed a higher GMV in the latero-medial temporal cortices bilaterally in the control and cognitively frail compared to $\mathrm{MCl}$ and Alzheimer's disease groups. Like the controls, the cognitively frail showed 
stronger associative and novelty deviant responses compared to $\mathrm{MCl}$ or Alzheimer's disease in relation to hippocampal and entorhinal volumes.

The cross-modal oddball task was designed to induce deviant responses from the superficial frontotemporal cortex, as neurophysiological markers of hippocampal-dependent associative learning. Alzheimer's patients show impairments in both sensory and associative memory, reduced medial temporal lobe activity to novelty (Sperling et al., 2003; Dickerson et al., 2005), and reduced electrophysiological response to oddballs (Engeland et al., 2002; Lee et al., 2013; Ruzzoli et al., 2016). We confirmed that Alzheimer's patients show reduced novelty and associative deviant responses. Neurophysiological profiles of the control and cognitively frail overlapped, and were significantly stronger compared to $\mathrm{MCl}$ and Alzheimer's disease groups. Task effects of the novelty deviant responses were observed across all regions of interest for the control and cognitively frail. The group-task interaction effects showing stronger associative and novelty deviant responses for the controls and cognitively frail were located in right Heschl's, bilateral superior temporal and inferior frontal gyri. In other words, the neuropsychologically impaired cognitively frail group does not show the neurophysiological signatures of early Alzheimer's disease. From a theoretical perspective, it is interesting to note that the response associated with associative deviants was weaker than novelty deviants, and did not differ qualitatively in terms of timing or distribution across our ROls. We had expected the associative deviant response to be more hippocampal-dependent, and hence more impaired with Alzheimer's pathology, but our current analyses suggest it was qualitatively similar to the more typical novelty deviant response.

To explore the differences between the community-based cognitively frail and Alzheimer's disease or $\mathrm{MCl}$, we tested volumetric differences in medial temporal lobe. Structurally, early Alzheimer's disease is characterised by atrophy in the medial temporal lobe as a function of tau burden (Braak et al., 2006; Schwarz et al., 2016). Recent studies of cognitive frailty have suggested frontotemporal and subcortical atrophy (Del Brutto et al., 2017; Gallucci et al., 2018), increased white matter hyperintensities (Avila-Funes et al., 2017; Del Brutto et al., 2017; Sugimoto et al., 2019), and decreased white matter microstructure integrity (Avila-Funes et al., 2017). We did not find structural differences between the control and cognitively frail in medial temporal lobe structures. The community-based groups showed significantly larger hippocampus and entorhinal volumes compared to patients with $\mathrm{MCl}$ and Alzheimer's disease, and did not show early structural signatures of Alzheimer's disease. The difference between our study and the previous work may lie in the epidemiological approach to baseline recruitment through the Cam-CAN 3000 cohort, rather than clinical referral pathways.

The neuropsychological profile of the cognitively frail resembled $\mathrm{MCl}$ group. They scored lower than the healthy controls on every ACER subscale. Compared to the $\mathrm{MCl}$ patients, they were more impaired on fluency, which might indicate an underlying executive deficit. Previous studies have suggested that the neuropsychological profile of cognitive frailty differs from $\mathrm{MCl}$ in episodic memory with domains of language, visuospatial skills and executive function relatively spared (Collie and Maruff, 2000). The cognitive impairment profile in frail adults has been described in terms of deficits in executive function and attention. Frail adults tend to not use cues effectively to retrieve stored information (Canevelli et al., 2015; Delrieu et al., 2016); have slower reaction times (O'Halloran et al., 2014; Robertson et al., 2014), show lower meta-cognitive awareness, and error monitoring (Amanzio et al., 2017).

However, many previous studies have focused on cognitive impairment in the context of physical frailty, rather than defining cognitive frailty in terms of poor cognitive function in a non-clinical community-dwelling cohort. The cognitive underperformance of our cognitively frail, might partly be attributed to their shorter education, coupled with a bias in most cognitive tests towards the 
better educated (Huppert et al., 2005). That is, highly educated individuals perform better on cognitive tests like MMSE and ACER, unless scores are normalised by education (Crane et al., 2006; Mathuranath et al., 2007; Amaral-Carvalho and Caramelli, 2012).

Our findings support the hypothesis that cognitive frailty represents part of the spectrum of normal neurocognitive function, rather than incipient Alzheimer's disease. This conclusion calls for a re-evaluation of the prior findings that associate cognitive frailty with higher incidence of dementia and faster cognitive decline (Buchman et al., 2007; Kojima et al., 2016; Shimada et al., 2018). These former studies have quantified the dementia incidence including all subtypes of dementia, however this association was highest in non-Alzheimer's dementias, particularly for vascular dementia (Panza et al., 2006; Avila-Funes et al., 2012; Gray et al., 2013; Aguilar-Navarro et al., 2016; Solfrizzi et al., 2017a). Although the link between cognitive frailty and Alzheimer's disease in previous studies is not conclusive, the two entities might share common risk factors such as cardiovascular disease (Panza et al., 2006; Frisoli et al., 2015; Fuhrmann et al., 2019) and hearing impairment (Valentijn et al., 2005; Panza et al., 2015).

In addition to the cardiovascular risk factors (Newman et al., 2001; Patrick et al., 2002; Fuhrmann et al., 2019), the cognitive underperformance of our cognitively frail group could be a result of cumulative effects of multiple psychosocial and medical risk factors. Malnutrition (Mulero et al., 2011; Chye et al., 2018; Rietman et al., 2018), social isolation (Robertson et al., 2013), sedentary lifestyle (Landi et al., 2010), lack of intellectual cognitive activities (Jung et al., 2010), psychiatric illnesses and long term use of antidepressants (Paulson and Lichtenberg, 2013; Gray et al., 2015), chronic inflammation (Weaver et al., 2002; Solfrizzi et al., 2017b) and lower education levels (Rogers et al., 2017) are known risk factors affecting healthy ageing. Here, the cognitively frail group had significantly lower education levels, compared to the controls and $\mathrm{MCl}$. This is a common pattern observed in other frailty studies (Brigola et al., 2019; Margioti et al., 2020). The cognitively frail population have significantly lower occurrence of third-level education (Robertson et al., 2014), and are twice as likely to have no educational qualifications (Rogers et al., 2017). Further, strong association between educational level and frailty was linked to mediating socioeconomic, behavioural, and psychosocial factors such as low income, chronic diseases, obesity, depression, unhealthy lifestyle, and chronic stress (Hoogendijk et al., 2014). This is consistent with the cognitive reserve hypothesis that an individual's prior education and cognitive abilities modify the resilience of brain structure to disease and injury (Stern, 2002). Longer education in early life and continuing diverse cognitive leisure activities in midlife and old age contribute to an individual's cognitive reserve, is related to better cognitive functioning in old age (Singh-Manoux et al., 2011; Borgeest et al., 2018; Lavrencic et al., 2018; Brigola et al., 2019) and having fewer symptoms of cognitive decline and neuropathology (Mortimer et al., 2003; Chapko et al., 2018).

The study has several limitations. Due to the cross-sectional design of the study we are unable to quantify the rates of progression or conversion to dementia from cognitive frailty. Longitudinal cognitive and neuroimaging studies would be useful to confirm the rate of conversion to Alzheimer's disease or other dementia, and potential mediators of conversion. Further, this study did not incorporate Alzheimer's disease biomarkers and instead used clinical criteria and neuropsychological criteria to define the groups. The cognitively frail group was defined using a standard threshold on ACER and MMSE. Future studies investigating the link between cognitive frailty and Alzheimer's disease may test for biomarkers of Alzheimer's such as tau and amyloidbeta measures acquired from blood, cerebrospinal fluid or positron emission tomography. Future studies may also assess the polygenic risk for Alzheimer's disease using common (e.g. APOE) and rare variants associated with the disease, which would help disentangle environmental and psychosocial risk factors from genetic risk factors contributing to cognitive frailty's aetiology. 
Further work is needed to clarify genetic and pathology-based features of cognitive frailty in relation to Alzheimer's disease and other dementias.

Our findings provide new evidence that community-dwelling cognitively frail older adults are neurophysiologically and structurally similar to those with more successful cognitive ageing, without the structural or neurophysiological features of $\mathrm{MCl}$ or Alzheimer's disease, despite similarly poor cognitive function to $\mathrm{MCl}$. Their underperformance on cognitive tests may be due to lower cognitive reserve and other risk factors across the lifespan. 


\section{References}

Aguilar-Navarro SG, Mimenza-Alvarado AJ, Anaya-Escamilla A, Gutiérrez-Robledo LM (2016) Frailty and Vascular Cognitive Impairment: Mechanisms Behind the Link. Rev Invest Clin 68:25-32.

Ahmed S, Mitchell J, Arnold R, Nestor PJ, Hodges JR (2008) Predicting rapid clinical progression in amnestic mild cognitive impairment. Dement Geriatr Cogn Disord 25:170-177.

Amanzio M, Palermo S, Zucca M, Rosato R, Rubino E, Leotta D, Bartoli M, Rainero I (2017) Neuropsychological Correlates of Pre-Frailty in Neurocognitive Disorders: A Possible Role for Metacognitive Dysfunction and Mood Changes. Front Med (Lausanne) 4:199.

Amaral-Carvalho V, Caramelli P (2012) Normative data for healthy middle-aged and elderly performance on the Addenbrooke Cognitive Examination-Revised. Cogn Behav Neurol 25:7276.

Ashburner J (2007) A fast diffeomorphic image registration algorithm. Neuroimage 38:95-113.

Ashburner J, Friston KJ (2005) Unified segmentation. Neuroimage 26:839-851.

Avila-Funes JA, Carcaillon L, Helmer C, Carrière I, Ritchie K, Rouaud O, Tzourio C, Dartigues JF, Amieva H (2012) Is frailty a prodromal stage of vascular dementia? Results from the Three-City Study. J Am Geriatr Soc 60:1708-1712.

Avila-Funes JA, Pelletier A, Meillon C, Catheline G, Periot O, Trevin O-Frenk I, Gonzalez-Colaço M, Dartigues JF, Pérès K, Allard M, Dilharreguy B, Amieva H (2017) Vascular Cerebral Damage in Frail Older Adults: The AMImage Study. J Gerontol A Biol Sci Med Sci 72:971-977.

Blackwell AD, Sahakian BJ, Vesey R, Semple JM, Robbins TW, Hodges JR (2004) Detecting dementia: novel neuropsychological markers of preclinical Alzheimer's disease. Dement Geriatr Cogn Disord 17:42-48.

Borgeest GS, Henson RN, Shafto M, Samu DC, Kievit R (2018) Greater lifestyle engagement is associated with better cognitive resilience. PsyArXiv.

Braak H, Alafuzoff I, Arzberger T, Kretzschmar H, Del Tredici K (2006) Staging of Alzheimer diseaseassociated neurofibrillary pathology using paraffin sections and immunocytochemistry. Acta Neuropathol 112:389-404.

Brigola AG, Alexandre TDS, Inouye K, Yassuda MS, Pavarini SCI, Mioshi E (2019) Limited formal education is strongly associated with lower cognitive status, functional disability and frailty status in older adults. Dement Neuropsychol 13:216-224.

Buchman AS, Boyle PA, Wilson RS, Tang Y, Bennett DA (2007) Frailty is associated with incident Alzheimer's disease and cognitive decline in the elderly. Psychosom Med 69:483-489.

Canevelli M, Cesari M, van Kan GA (2015) Frailty and cognitive decline: how do they relate? Curr Opin Clin Nutr Metab Care 18:43-50.

Cappola AR, Xue QL, Ferrucci L, Guralnik JM, Volpato S, Fried LP (2003) Insulin-like growth factor I and interleukin-6 contribute synergistically to disability and mortality in older women. J Clin Endocrinol Metab 88:2019-2025.

Chapko D, McCormack R, Black C, Staff R, Murray A (2018) Life-course determinants of cognitive reserve (CR) in cognitive aging and dementia - a systematic literature review. Aging Ment Health 22:915-926.

Chye L, Wei K, Nyunt MSZ, Gao Q, Wee SL, Ng TP (2018) Strong Relationship between Malnutrition and Cognitive Frailty in the Singapore Longitudinal Ageing Studies (SLAS-1 and SLAS-2). J Prev Alzheimers Dis 5:142-148.

Collie A, Maruff P (2000) The neuropsychology of preclinical Alzheimer's disease and mild cognitive impairment. Neurosci Biobehav Rev 24:365-374.

Coquelet N, Mary A, Peigneux P, Goldman S, Wens V, De Tiège X (2017) The electrophysiological connectome is maintained in healthy elders: a power envelope correlation MEG study. Sci Rep 7:13984.

Crane PK, Gibbons LE, Jolley L, van Belle G, Selleri R, Dalmonte E, De Ronchi D (2006) Differential item functioning related to education and age in the Italian version of the Mini-mental State Examination. Int Psychogeriatr 18:505-515. 
de Haan W, van der Flier WM, Wang H, Van Mieghem PF, Scheltens P, Stam CJ (2012) Disruption of functional brain networks in Alzheimer's disease: what can we learn from graph spectral analysis of resting-state magnetoencephalography? Brain Connect 2:45-55.

de Rover M, Pironti VA, McCabe JA, Acosta-Cabronero J, Arana FS, Morein-Zamir S, Hodges JR, Robbins TW, Fletcher PC, Nestor PJ, Sahakian BJ (2011) Hippocampal dysfunction in patients with mild cognitive impairment: a functional neuroimaging study of a visuospatial paired associates learning task. Neuropsychologia 49:2060-2070.

Del Brutto OH, Mera RM, Cagino K, Fanning KD, Milla-Martinez MF, Nieves JL, Zambrano M, Sedler MJ (2017) Neuroimaging signatures of frailty: A population-based study in community-dwelling older adults (the Atahualpa Project). Geriatr Gerontol Int 17:270-276.

Delorme A, Makeig S (2004) EEGLAB: an open source toolbox for analysis of single-trial EEG dynamics including independent component analysis. J Neurosci Methods 134:9-21.

Delrieu J, Andrieu S, Pahor M, Cantet C, Cesari M, Ousset PJ, Voisin T, Fougère B, Gillette S, Carrie I, Vellas B (2016) Neuropsychological Profile of "Cognitive Frailty" Subjects in MAPT Study. J Prev Alzheimers Dis 3:151-159.

Dickerson BC, Salat DH, Greve DN, Chua EF, Rand-Giovannetti E, Rentz DM, Bertram L, Mullin K, Tanzi RE, Blacker D, Albert MS, Sperling RA (2005) Increased hippocampal activation in mild cognitive impairment compared to normal aging and AD. Neurology 65:404-411.

Engeland C, Mahoney C, Mohr E, llivitsky V, Knott VJ (2002) Acute nicotine effects on auditory sensory memory in tacrine-treated and nontreated patients with Alzheimer's disease: an eventrelated potential study. Pharmacol Biochem Behav 72:457-464.

Frisoli A, Ingham SJ, Paes Â, Tinoco E, Greco A, Zanata N, Pintarelli V, Elber I, Borges J, Camargo Carvalho AC (2015) Frailty predictors and outcomes among older patients with cardiovascular disease: Data from Fragicor. Arch Gerontol Geriatr 61:1-7.

Fuhrmann D, Nesbitt D, Shafto M, Rowe JB, Price D, Gadie A, Kievit RA, Cam-CAN (2019) Strong and specific associations between cardiovascular risk factors and white matter micro- and macrostructure in healthy aging. Neurobiol Aging 74:46-55.

Gallucci M, Piovesan C, Di Battista ME (2018) Associations between the Frailty Index and Brain Atrophy: The Treviso Dementia (TREDEM) Registry. J Alzheimers Dis 62:1623-1634.

Garrido MI, Kilner JM, Stephan KE, Friston KJ (2009) The mismatch negativity: a review of underlying mechanisms. Clin Neurophysiol 120:453-463.

Ge ML, Carlson MC, Bandeen-Roche K, Chu NM, Tian J, Kasper JD, Xue QL (2020) U.S. National Profile of Older Adults with Cognitive Impairment Alone, Physical Frailty Alone, and Both. J Am Geriatr Soc 68:2822-2830.

Goldenholz DM, Ahlfors SP, Hämäläinen MS, Sharon D, Ishitobi M, Vaina LM, Stufflebeam SM (2009) Mapping the signal-to-noise-ratios of cortical sources in magnetoencephalography and electroencephalography. Hum Brain Mapp 30:1077-1086.

Gottfried JA, Dolan RJ (2003) The nose smells what the eye sees: crossmodal visual facilitation of human olfactory perception. Neuron 39:375-386.

Gray SL, Anderson ML, Hubbard RA, LaCroix A, Crane PK, McCormick W, Bowen JD, McCurry SM, Larson EB (2013) Frailty and incident dementia. J Gerontol A Biol Sci Med Sci 68:1083-1090.

Gray SL, Anderson ML, Dublin S, Hanlon JT, Hubbard R, Walker R, Yu O, Crane PK, Larson EB (2015) Cumulative use of strong anticholinergics and incident dementia: a prospective cohort study. JAMA Intern Med 175:401-407.

Hardy J, Selkoe DJ (2002) The amyloid hypothesis of Alzheimer's disease: progress and problems on the road to therapeutics. Science 297:353-356.

Harrison TM, Burggren AC, Small GW, Bookheimer SY (2016) Altered memory-related functional connectivity of the anterior and posterior hippocampus in older adults at increased genetic risk for Alzheimer's disease. Hum Brain Mapp 37:366-380.

Henson RN, Mouchlianitis E, Friston KJ (2009) MEG and EEG data fusion: simultaneous localisation of face-evoked responses. Neuroimage 47:581-589. 
Hoogendijk EO, van Hout HP, Heymans MW, van der Horst HE, Frijters DH, Broese van Groenou MI, Deeg DJ, Huisman M (2014) Explaining the association between educational level and frailty in older adults: results from a 13-year longitudinal study in the Netherlands. Ann Epidemiol 24:538-544.e532.

Hughes LE, Rowe JB (2013) The impact of neurodegeneration on network connectivity: a study of change detection in frontotemporal dementia. J Cogn Neurosci 25:802-813.

Hughes LE, Rittman T, Robbins TW, Rowe JB (2018) Reorganization of cortical oscillatory dynamics underlying disinhibition in frontotemporal dementia. Brain 141:2486-2499.

Hughes LE, Henson RN, Pereda E, Bruña R, López-Sanz D, Quinn AJ, Woolrich MW, Nobre AC, Rowe JB, Maestú F, Group BW (2019) Biomagnetic biomarkers for dementia: A pilot multicentre study with a recommended methodological framework for magnetoencephalography. Alzheimers Dement (Amst) 11:450-462.

Huppert FA, Cabelli ST, Matthews FE, Study MCFaA (2005) Brief cognitive assessment in a UK population sample -- distributional properties and the relationship between the MMSE and an extended mental state examination. BMC Geriatr 5:7.

Joassin F, Pesenti M, Maurage P, Verreckt E, Bruyer R, Campanella S (2011) Cross-modal interactions between human faces and voices involved in person recognition. Cortex 47:367-376.

Jung Y, Gruenewald TL, Seeman TE, Sarkisian CA (2010) Productive activities and development of frailty in older adults. J Gerontol B Psychol Sci Soc Sci 65B:256-261.

Kelaiditi E, Cesari M, Canevelli M, van Kan GA, Ousset PJ, Gillette-Guyonnet S, Ritz P, Duveau F, Soto ME, Provencher V, Nourhashemi F, Salvà A, Robert P, Andrieu S, Rolland Y, Touchon J, Fitten JL, Vellas B, IANA/IAGG (2013) Cognitive frailty: rational and definition from an (I.A.N.A./I.A.G.G.) international consensus group.J Nutr Health Aging 17:726-734.

Kocagoncu E, Quinn A, Firouzian A, Cooper E, Greve A, Gunn R, Green G, Woolrich MW, Henson RN, Lovestone S, Rowe JB, team DaFPs (2020) Tau pathology in early Alzheimer's disease is linked to selective disruptions in neurophysiological network dynamics. Neurobiol Aging 92:141-152.

Kojima G, Taniguchi Y, lliffe S, Walters K (2016) Frailty as a Predictor of Alzheimer Disease, Vascular Dementia, and All Dementia Among Community-Dwelling Older People: A Systematic Review and Meta-Analysis. J Am Med Dir Assoc 17:881-888.

LaFerla FM, Oddo S (2005) Alzheimer's disease: Abeta, tau and synaptic dysfunction. Trends Mol Med 11:170-176.

Landi F, Abbatecola AM, Provinciali M, Corsonello A, Bustacchini S, Manigrasso L, Cherubini A, Bernabei R, Lattanzio F (2010) Moving against frailty: does physical activity matter? Biogerontology 11:537-545.

Langlois F, Vu TT, Kergoat MJ, Chassé K, Dupuis G, Bherer L (2012) The multiple dimensions of frailty: physical capacity, cognition, and quality of life. Int Psychogeriatr 24:1429-1436.

Laptinskaya D, Thurm F, Küster OC, Fissler P, Schlee W, Kolassa S, von Arnim CAF, Kolassa IT (2018) Auditory Memory Decay as Reflected by a New Mismatch Negativity Score Is Associated with Episodic Memory in Older Adults at Risk of Dementia. Front Aging Neurosci 10:5.

Lavrencic LM, Richardson C, Harrison SL, Muniz-Terrera G, Keage HAD, Brittain K, Kirkwood TBL, Jagger C, Robinson L, Stephan BCM (2018) Is There a Link Between Cognitive Reserve and Cognitive Function in the Oldest-Old? J Gerontol A Biol Sci Med Sci 73:499-505.

Lee MS, Lee SH, Moon EO, Moon YJ, Kim S, Kim SH, Jung IK (2013) Neuropsychological correlates of the P300 in patients with Alzheimer's disease. Prog Neuropsychopharmacol Biol Psychiatry 40:62-69.

Maestú F, Peña JM, Garcés P, González S, Bajo R, Bagic A, Cuesta P, Funke M, Mäkelä JP, Menasalvas E, Nakamura A, Parkkonen L, López ME, Del Pozo F, Sudre G, Zamrini E, Pekkonen E, Henson RN, Becker JT, Disease MICoAs (2015) A multicenter study of the early detection of synaptic dysfunction in Mild Cognitive Impairment using Magnetoencephalography-derived functional connectivity. Neuroimage Clin 9:103-109. 
Margioti E, Kosmidis MH, Yannakoulia M, Dardiotis E, Hadjigeorgiou G, Sakka P, Ntanasi E, Vlachos GS, Scarmeas N (2020) Exploring the association between subjective cognitive decline and frailty: the Hellenic Longitudinal Investigation of Aging and Diet Study (HELIAD). Aging Ment Health 24:137-147.

Mathuranath PS, Cherian JP, Mathew R, George A, Alexander A, Sarma SP (2007) Mini mental state examination and the Addenbrooke's cognitive examination: effect of education and norms for a multicultural population. Neurol India 55:106-110.

McKhann GM, Knopman DS, Chertkow H, Hyman BT, Jack CR, Kawas CH, Klunk WE, Koroshetz WJ, Manly JJ, Mayeux R, Mohs RC, Morris JC, Rossor MN, Scheltens P, Carrillo MC, Thies B, Weintraub S, Phelps CH (2011) The diagnosis of dementia due to Alzheimer's disease: recommendations from the National Institute on Aging-Alzheimer's Association workgroups on diagnostic guidelines for Alzheimer's disease. Alzheimers Dement 7:263-269.

Mortimer JA, Snowdon DA, Markesbery WR (2003) Head circumference, education and risk of dementia: findings from the Nun Study. J Clin Exp Neuropsychol 25:671-679.

Mulero J, Zafrilla P, Martinez-Cacha A (2011) Oxidative stress, frailty and cognitive decline. J Nutr Health Aging 15:756-760.

Newman AB, Gottdiener JS, Mcburnie MA, Hirsch CH, Kop WJ, Tracy R, Walston JD, Fried LP, Group CHSR (2001) Associations of subclinical cardiovascular disease with frailty. J Gerontol A Biol Sci Med Sci 56:M158-166.

O'Halloran AM, Finucane C, Savva GM, Robertson IH, Kenny RA (2014) Sustained attention and frailty in the older adult population. J Gerontol B Psychol Sci Soc Sci 69:147-156.

Osipova D, Ahveninen J, Jensen O, Ylikoski A, Pekkonen E (2005) Altered generation of spontaneous oscillations in Alzheimer's disease. Neuroimage 27:835-841.

Panza F, Solfrizzi V, Logroscino G (2015) Age-related hearing impairment-a risk factor and frailty marker for dementia and AD. Nat Rev Neurol 11:166-175.

Panza F, D'Introno A, Colacicco AM, Capurso C, Parigi AD, Capurso SA, Caselli RJ, Pilotto A, Scafato E, Capurso A, Solfrizzi V (2006) Cognitive frailty: Predementia syndrome and vascular risk factors. Neurobiol Aging 27:933-940.

Patrick L, Gaskovski P, Rexroth D (2002) Cumulative illness and neuropsychological decline in hospitalized geriatric patients. Clin Neuropsychol 16:145-156.

Paulson D, Lichtenberg PA (2013) Vascular depression: an early warning sign of frailty. Aging Ment Health 17:85-93.

Pekkonen E (2000) Mismatch negativity in aging and in Alzheimer's and Parkinson's diseases. Audiol Neurootol 5:216-224.

Petersen RC, Caracciolo B, Brayne C, Gauthier S, Jelic V, Fratiglioni L (2014) Mild cognitive impairment: a concept in evolution. J Intern Med 275:214-228.

Phillips HN, Blenkmann A, Hughes LE, Kochen S, Bekinschtein TA, Cam-Can, Rowe JB (2016) Convergent evidence for hierarchical prediction networks from human electrocorticography and magnetoencephalography. Cortex 82:192-205.

Price D, Tyler LK, Neto Henriques R, Campbell KL, Williams N, Treder MS, Taylor JR, Henson RNA, CamCAN (2017) Age-related delay in visual and auditory evoked responses is mediated by whiteand grey-matter differences. Nat Commun 8:15671.

Rietman ML, van der A DL, van Oostrom SH, Picavet HSJ, Dollé MET, van Steeg H, Verschuren WMM, Spijkerman AMW (2018) The Association between BMI and Different Frailty Domains: A UShaped Curve? J Nutr Health Aging 22:8-15.

Robertson DA, Savva GM, Kenny RA (2013) Frailty and cognitive impairment--a review of the evidence and causal mechanisms. Ageing Res Rev 12:840-851.

Robertson DA, Savva GM, Coen RF, Kenny RA (2014) Cognitive function in the prefrailty and frailty syndrome. J Am Geriatr Soc 62:2118-2124.

Rogers NT, Steptoe A, Cadar D (2017) Frailty is an independent predictor of incident dementia: Evidence from the English Longitudinal Study of Ageing. Sci Rep 7:15746. 
Ruzzoli M, Pirulli C, Mazza V, Miniussi C, Brignani D (2016) The mismatch negativity as an index of cognitive decline for the early detection of Alzheimer's disease. Sci Rep 6:33167.

Schwarz AJ, Yu P, Miller BB, Shcherbinin S, Dickson J, Navitsky M, Joshi AD, Devous MD, Mintun MS (2016) Regional profiles of the candidate tau PET ligand 18F-AV-1451 recapitulate key features of Braak histopathological stages. Brain 139:1539-1550.

Shafto MA, Tyler LK, Dixon M, Taylor JR, Rowe JB, Cusack R, Calder AJ, Marslen-Wilson WD, Duncan J, Dalgleish T, Henson RN, Brayne C, Matthews FE, Cam-CAN (2014) The Cambridge Centre for Ageing and Neuroscience (Cam-CAN) study protocol: a cross-sectional, lifespan, multidisciplinary examination of healthy cognitive ageing. BMC Neurol 14:204.

Shimada H, Makizako H, Tsutsumimoto K, Doi T, Lee S, Suzuki T (2018) Cognitive Frailty and Incidence of Dementia in Older Persons. J Prev Alzheimers Dis 5:42-48.

Shimada H, Makizako H, Lee S, Doi T, Tsutsumimoto K, Harada K, Hotta R, Bae S, Nakakubo S, Suzuki T (2016) Impact of Cognitive Frailty on Daily Activities in Older Persons. J Nutr Health Aging 20:729-735.

Singh-Manoux A, Marmot MG, Glymour M, Sabia S, Kivimäki M, Dugravot A (2011) Does cognitive reserve shape cognitive decline? Ann Neurol 70:296-304.

Solfrizzi V, Scafato E, Frisardi V, Sancarlo D, Seripa D, Logroscino G, Baldereschi M, Crepaldi G, Di Carlo A, Galluzzo L, Gandin C, Inzitari D, Maggi S, Pilotto A, Panza F, Group ILSoAW (2012) Frailty syndrome and all-cause mortality in demented patients: the ftalian Longitudinal Study on Aging. Age (Dordr) 34:507-517.

Solfrizzi V, Scafato E, Seripa D, Lozupone M, Imbimbo BP, D'Amato A, Tortelli R, Schilardi A, Galluzzo L, Gandin C, Baldereschi M, Di Carlo A, Inzitari D, Daniele A, Sabbà C, Logroscino G, Panza F, Group ILSoAW (2017a) Reversible Cognitive Frailty, Dementia, and All-Cause Mortality. The Italian Longitudinal Study on Aging. J Am Med Dir Assoc 18:89.e81-89.e88.

Solfrizzi V, Scafato E, Lozupone M, Seripa D, Giannini M, Sardone R, Bonfiglio C, Abbrescia DI, Galluzzo L, Gandin C, Baldereschi M, Di Carlo A, Inzitari D, Daniele A, Sabbà C, Logroscino G, Panza F, Group ILSoAW (2017b) Additive Role of a Potentially Reversible Cognitive Frailty Model and Inflammatory State on the Risk of Disability: The Italian Longitudinal Study on Aging. Am J Geriatr Psychiatry 25:1236-1248.

Sperling RA, Bates JF, Chua EF, Cocchiarella AJ, Rentz DM, Rosen BR, Schacter DL, Albert MS (2003) fMRI studies of associative encoding in young and elderly controls and mild Alzheimer's disease. J Neurol Neurosurg Psychiatry 74:44-50.

Stam CJ, Jones BF, Manshanden I, van Cappellen van Walsum AM, Montez T, Verbunt JP, de Munck JC, van Dijk BW, Berendse HW, Scheltens P (2006) Magnetoencephalographic evaluation of resting-state functional connectivity in Alzheimer's disease. Neuroimage 32:1335-1344.

Stern Y (2002) What is cognitive reserve? Theory and research application of the reserve concept. J Int Neuropsychol Soc 8:448-460.

Sugimoto T, Ono R, Kimura A, Saji N, Niida S, Toba K, Sakurai T (2019) Cross-Sectional Association Between Cognitive Frailty and White Matter Hyperintensity Among Memory Clinic Patients. J Alzheimers Dis 72:605-612.

Talegawkar SA, Bandinelli S, Bandeen-Roche K, Chen P, Milaneschi Y, Tanaka T, Semba RD, Guralnik JM, Ferrucci L (2012) A higher adherence to a Mediterranean-style diet is inversely associated with the development of frailty in community-dwelling elderly men and women. J Nutr 142:2161-2166.

Tsvetanov KA, Henson RN, Tyler LK, Davis SW, Shafto MA, Taylor JR, Williams N, Cam-Can, Rowe JB (2015) The effect of ageing on $\mathrm{fMRI}$ : Correction for the confounding effects of vascular reactivity evaluated by joint fMRI and MEG in 335 adults. Hum Brain Mapp 36:2248-2269.

Valentijn SA, van Boxtel MP, van Hooren SA, Bosma H, Beckers HJ, Ponds RW, Jolles J (2005) Change in sensory functioning predicts change in cognitive functioning: results from a 6-year follow-up in the maastricht aging study. J Am Geriatr Soc 53:374-380. 
Vlahou EL, Thurm F, Kolassa IT, Schlee W (2014) Resting-state slow wave power, healthy aging and cognitive performance. Sci Rep 4:5101.

Walston J, McBurnie MA, Newman A, Tracy RP, Kop WJ, Hirsch CH, Gottdiener J, Fried LP, Study CH (2002) Frailty and activation of the inflammation and coagulation systems with and without clinical comorbidities: results from the Cardiovascular Health Study. Arch Intern Med 162:2333-2341.

Weaver JD, Huang MH, Albert M, Harris T, Rowe JW, Seeman TE (2002) Interleukin-6 and risk of cognitive decline: MacArthur studies of successful aging. Neurology 59:371-378.

West S, Bhugra P (2015) Emerging drug targets for $A \beta$ and tau in Alzheimer's disease: a systematic review. Br J Clin Pharmacol 80:221-234. 


\section{Figures and captions}

A

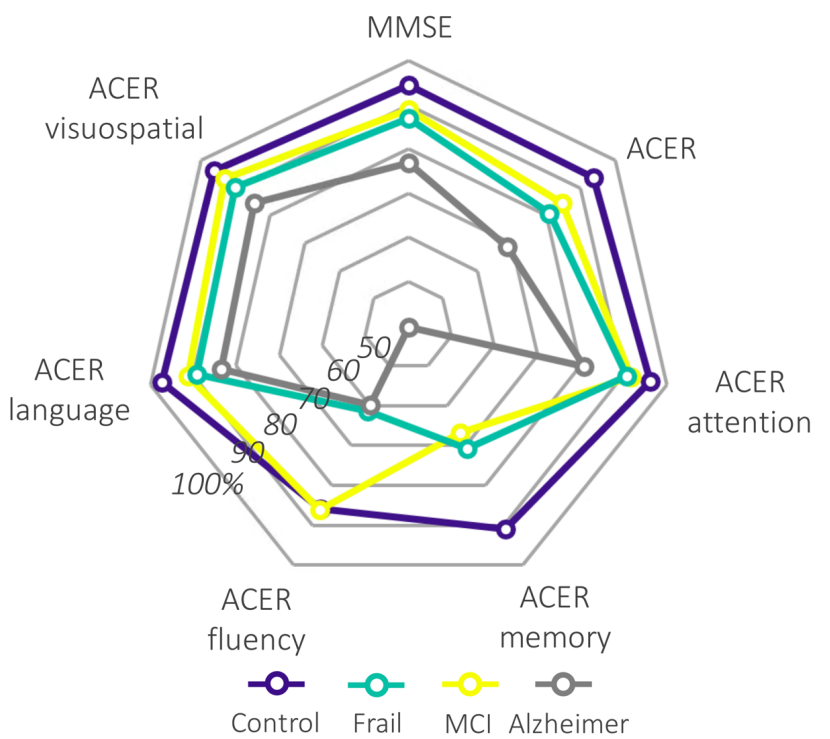

C

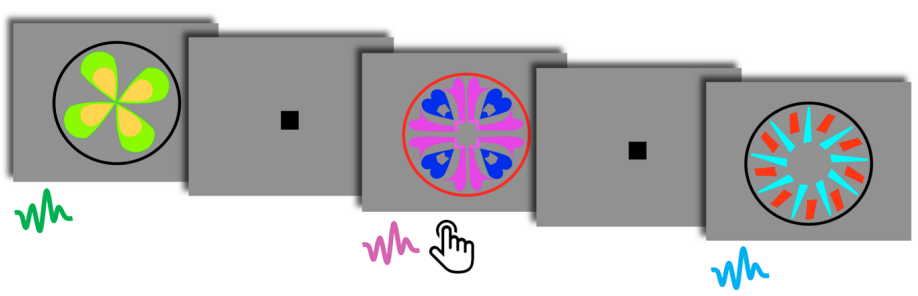

B
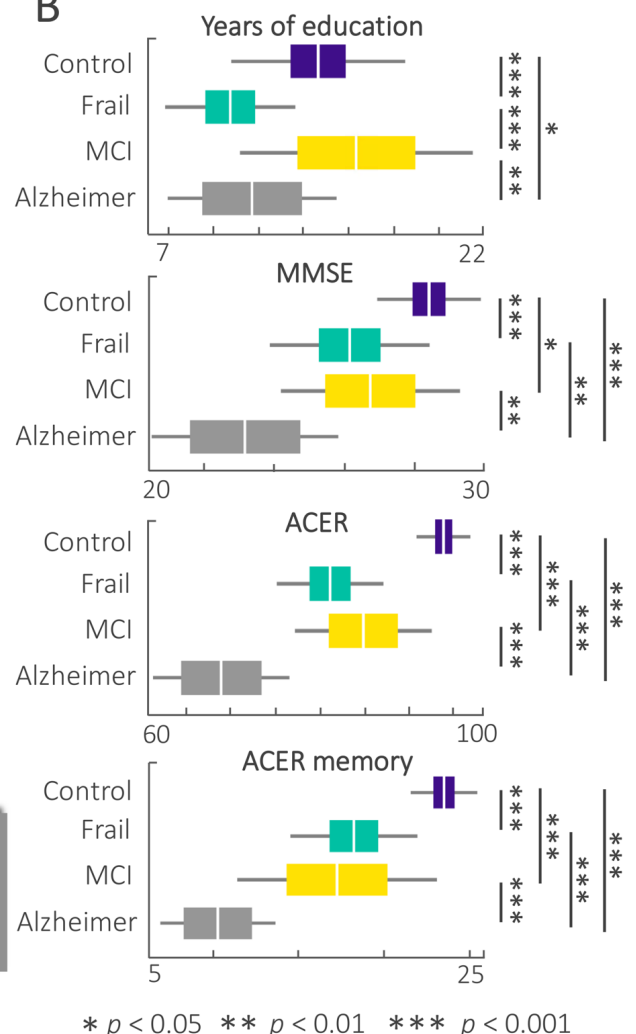

$* p<0.05 * * p<0.01 * * * p<0.001$

Figure 1. A. The radar chart displays the group means for the neuropsychological tests converted to percentages against the maximum score in each test for ease of comparison across groups. Note that the performance of the cognitively frail group overlaps with the $\mathrm{MCl}$ across all tests except for ACER fluency. B. Group differences in education levels and neuropsychological tests. The cognitively frail group had lower education levels than the controls. On the neuropsychological tests, the cognitively frail performed similar to the $\mathrm{MCl}$ group. C. Example stimuli from the cross-modal oddball task. The images were presented together with paired sounds after the $300 \mathrm{~ms}$ lag. Participants were asked to press the button whenever they saw a red circle around the image. $\mathrm{MCl}$ : Symptomatic mild cognitive impairment after secondary/tertiary memory clinic assessment. 


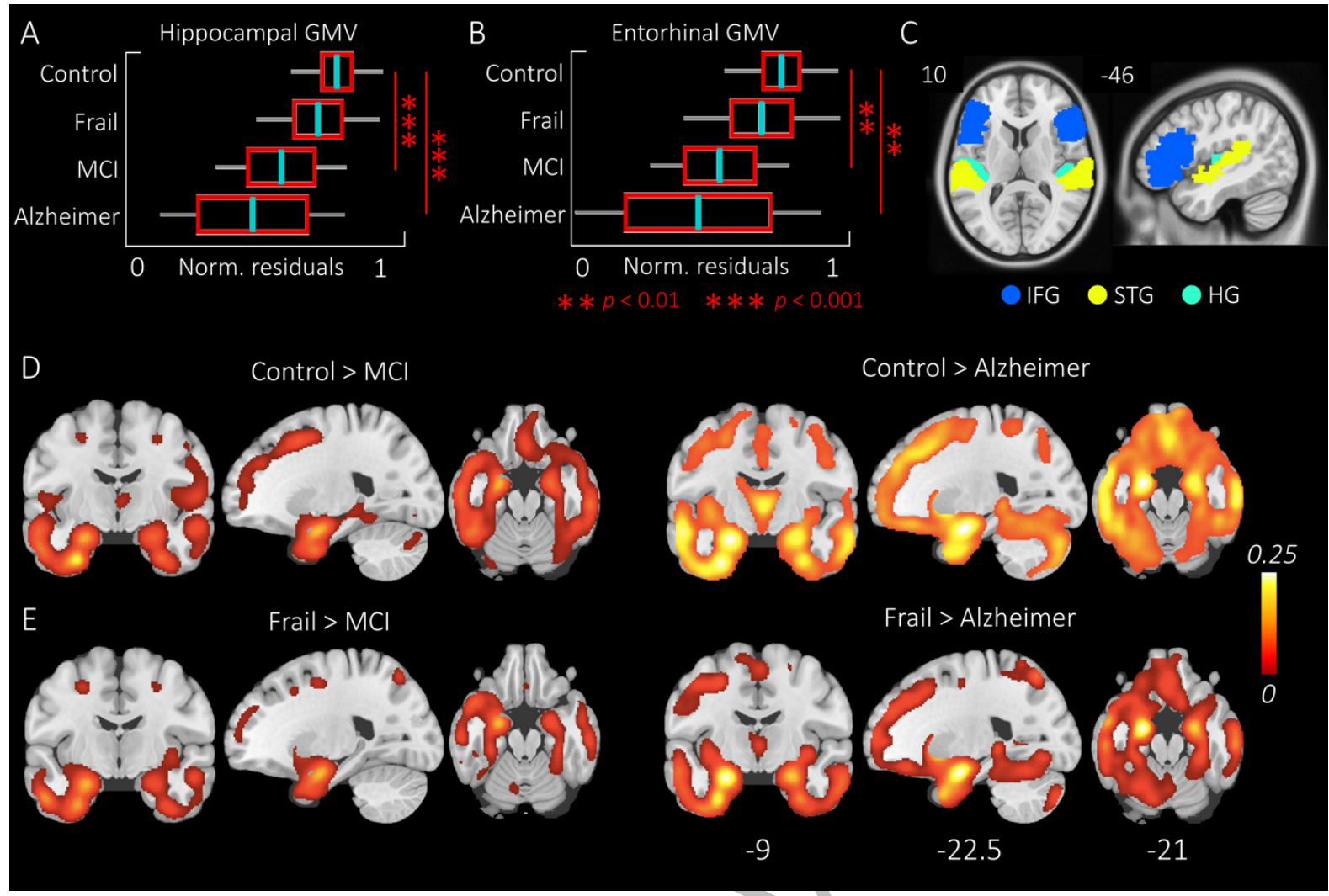

Figure 2. A-B. GMV differences across groups in the hippocampus and entorhinal cortex. The boxplots display the normalised residuals after correcting for differences in age and total intracranial volume (TIV). There were no significant differences in volume between the cognitively frail and the control group. C. 6 ROIs used in the RMS analysis. ROls comprise inferior frontal gyri (IFG), superior temporal gyri (STG) and Heschl's gyri (HG) bilaterally. D-E. The contrast images from the VBM analysis. Control and the cognitively frail show similar patterns of grey matter volume compared to the $\mathrm{MCl}$ and Alzheimer's disease groups. See the Extended data for the grey matter volume analysis of the lateral frontotemporal ROls used in the RMS analysis (Figure 2-1). $\mathrm{MCl}$ : Symptomatic mild cognitive impairment after secondary/tertiary memory clinic assessment. 


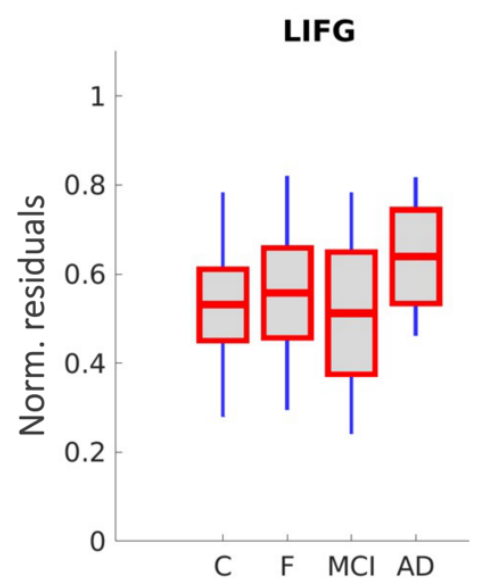

RIFG
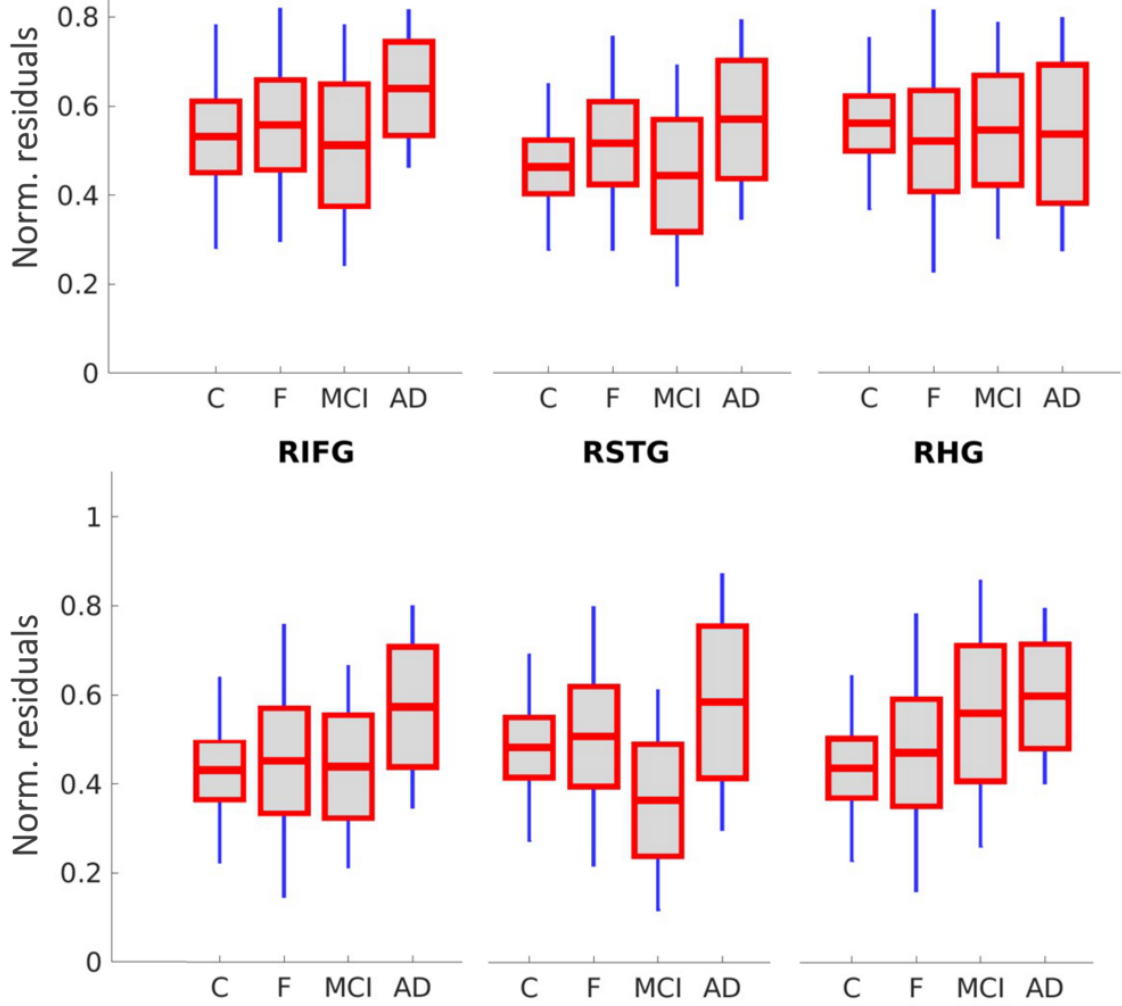

Figure 2-1: Grey matter volume analysis of the lateral frontotemporal network. In Alzheimer's disease. atrophy of the auditory cortex comes at the later isocortical Braak stages (ie V-VI) along with other neocortical areas such as the frontal cortex. Therefore, in the early stages of Alzheimer's disease, the integrity of the auditory cortex is not expected to be compromised, and consequently, diminished deviant responses observed in our clinical population are not expected be attributed to the atrophied auditory cortex. To provide support for this claim, we performed the confirmatory grey matter volume comparisons across the four groups within the lateral frontotemporal ROls which were used to extract neurophysiological signals from. We performed ANCOVAs to test for group differences whilst accounting for differences in age and TIV. As expected there were no significant effect of the group in any of the six ROIs, confirming that the differences we find in the deviant responses could not be attributed to local GM atrophy. C: Controls; F: Cognitively frail; MCl: Mild cognitive impairment; AD: Alzheimer's disease. 
A

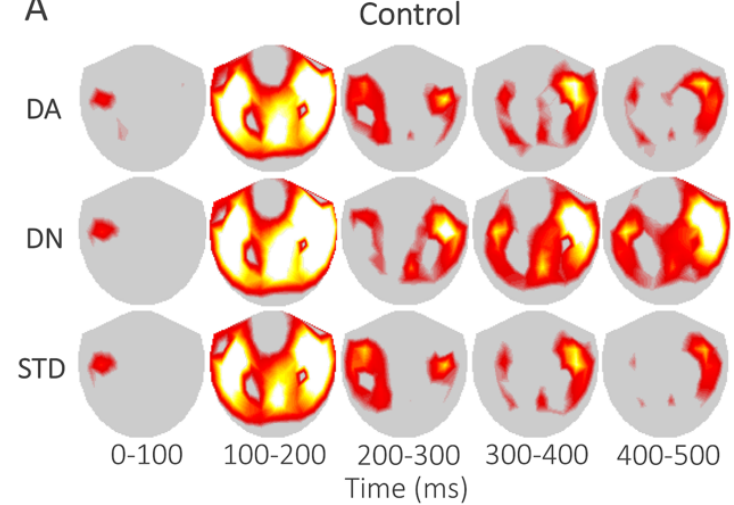

C

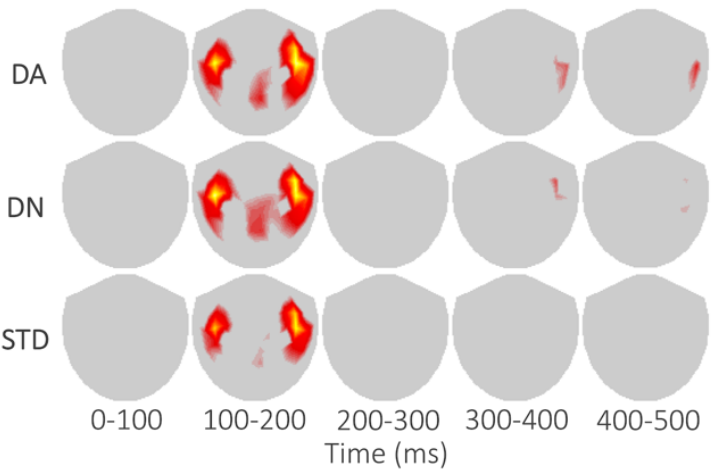

B

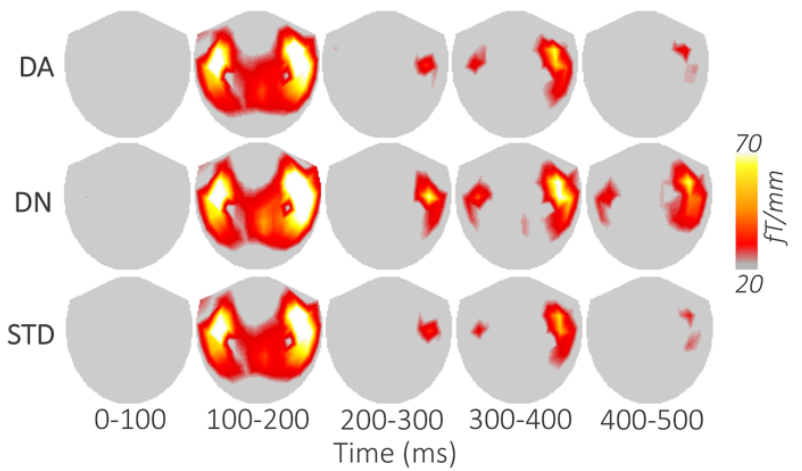

D

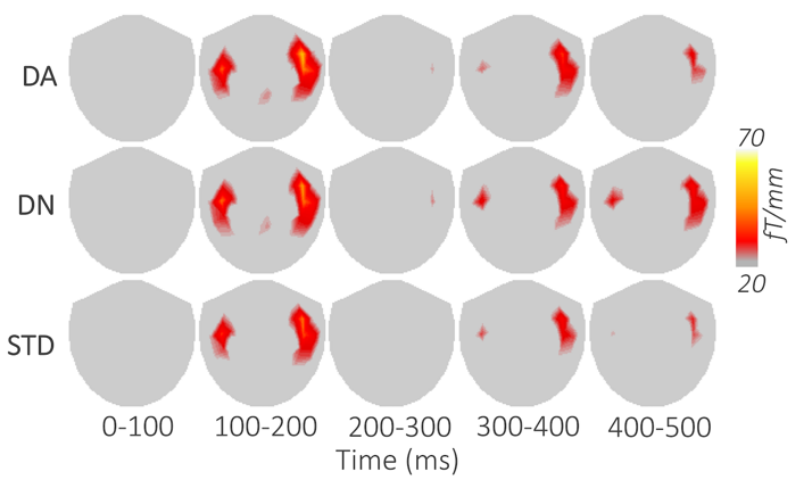

E
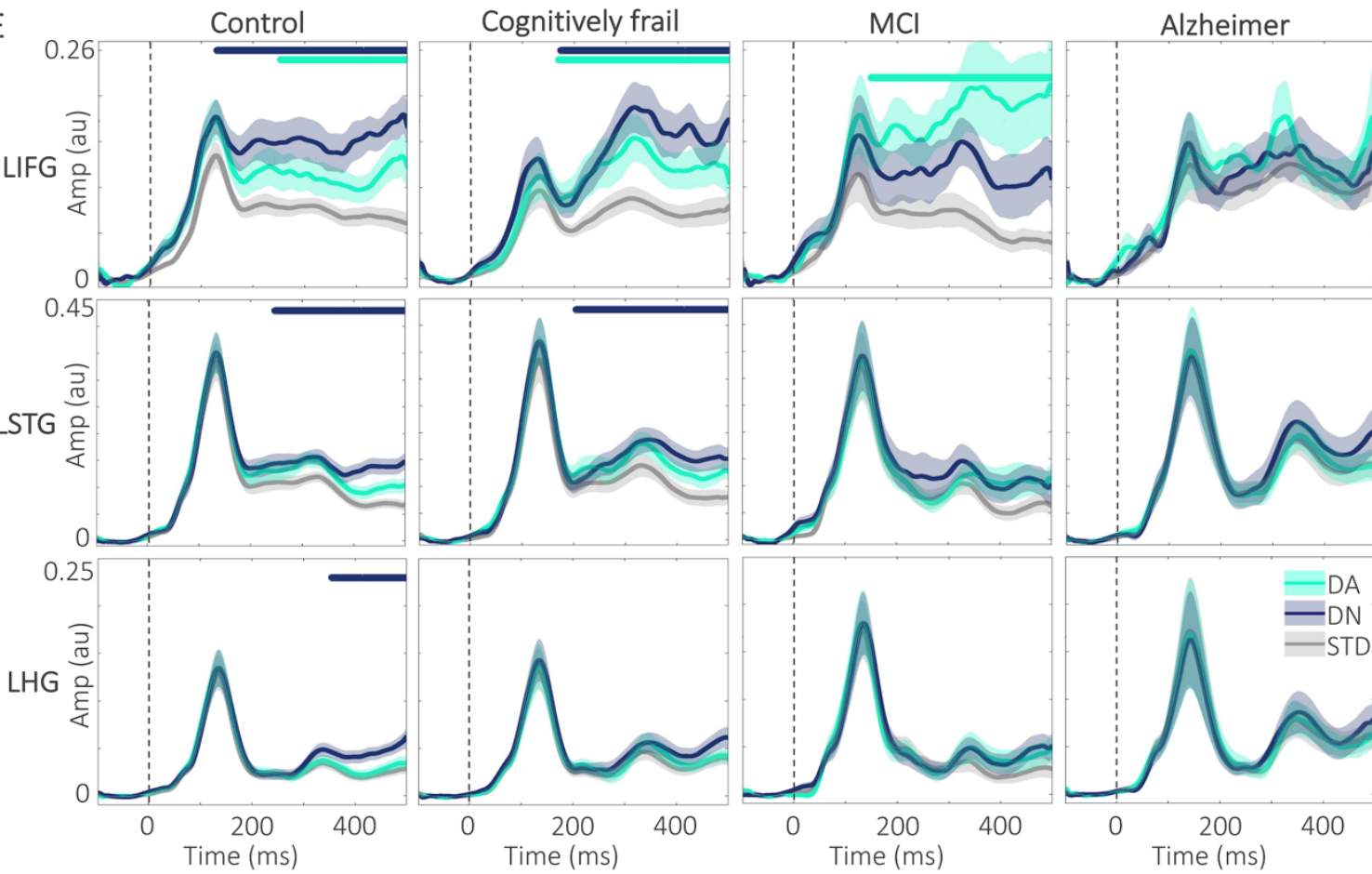

Figure 3. Associative and novelty deviant responses by group. A-D. Topoplots show the mean gradiometer activity across the scalp for the DA, DN and STD conditions in $100 \mathrm{~ms}$ time windows in four groups. Note that the DN amplitude is higher after the N100 peak, compared to both DA and STD conditions in control and cognitively frail groups. The gradiometer activity in the $\mathrm{MCl}$ and Alzheimer's disease groups are weaker compared to the control and cognitively frail groups. E. Plots display the RMS time series for the left hemisphere ROIs for simplicity. Dashed vertical lines mark the sound onset. Note that the amplitude differences between the deviants and the standard in the frontal regions were larger than the temporal regions, and the deviant effects are stronger in the control and cognitively frail groups; and that there is considerably higher variance in 
the $\mathrm{MCl}$ group. See the Extended data for the renderings of the source activity (Figure 3-1). Amp: Amplitude; AU: Arbitrary units; DA: Associative deviant; DN: Novelty deviant; fT: Femtotesla; LHG: Left Heschl's gyrus; LIFG: Left inferior frontal gyrus; LSTG: Left superior temporal gyrus; STD: Standard. 


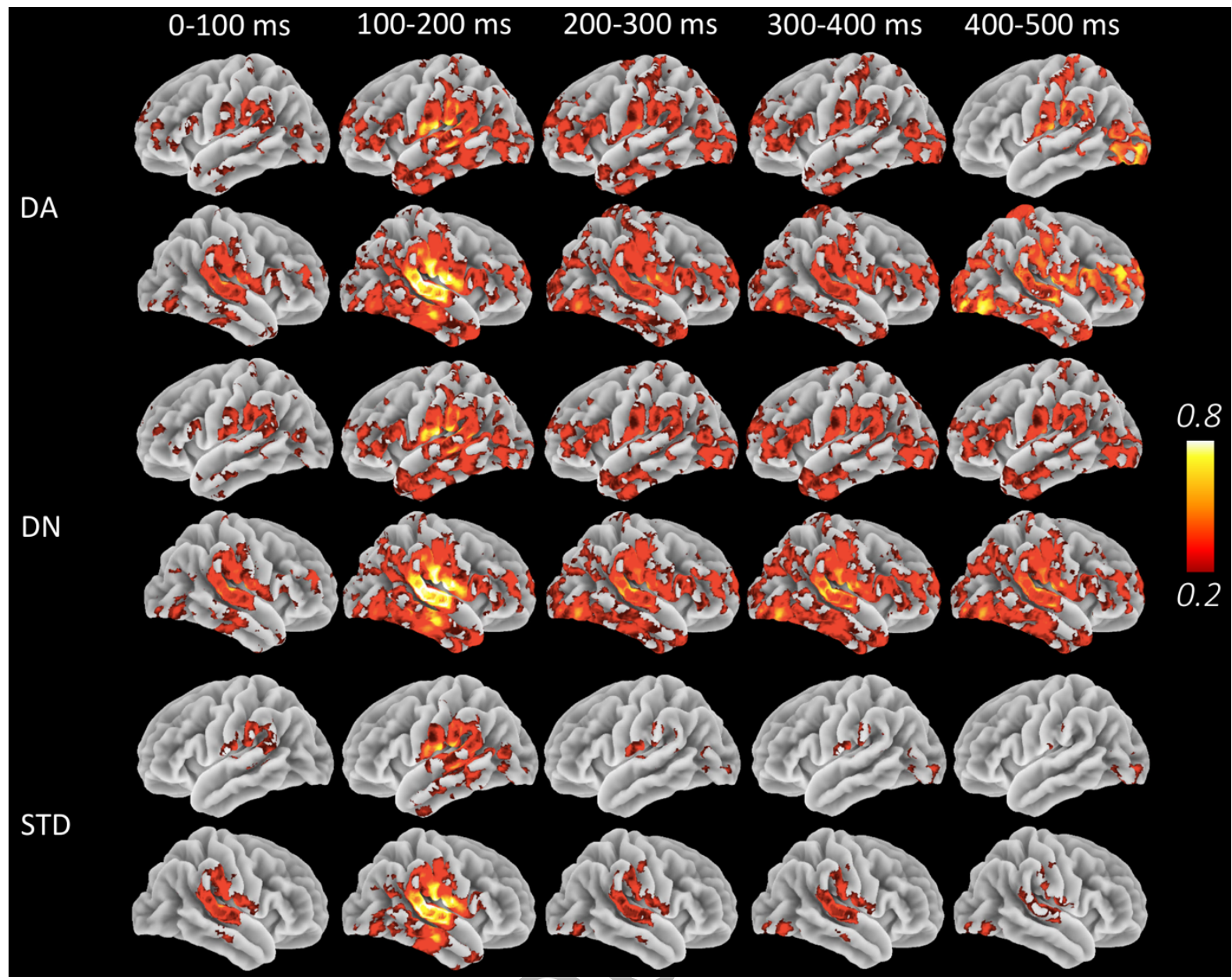

Figure 3-1: Source activity: The renderings display mean source activity in 100 ms moving time windows in the Control group. In line with the topoplots activity and our RMS findings, here we find the activation of a bilateral frontotemporal network peaking at $100 \mathrm{~ms}$. Further compared to the standard condition, deviant conditions show stronger and more widespread activity in the frontotemporal regions $200 \mathrm{~ms}$ after the sound onset. DA: Associative deviant; DN: Novelty deviant: STD: Standard. 
A

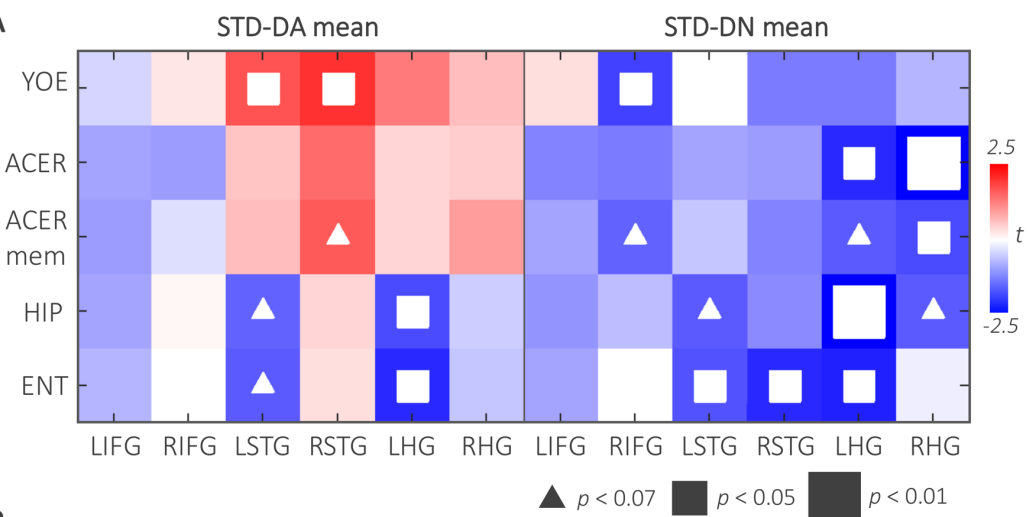

B

STD-DA mean $x$ HIP
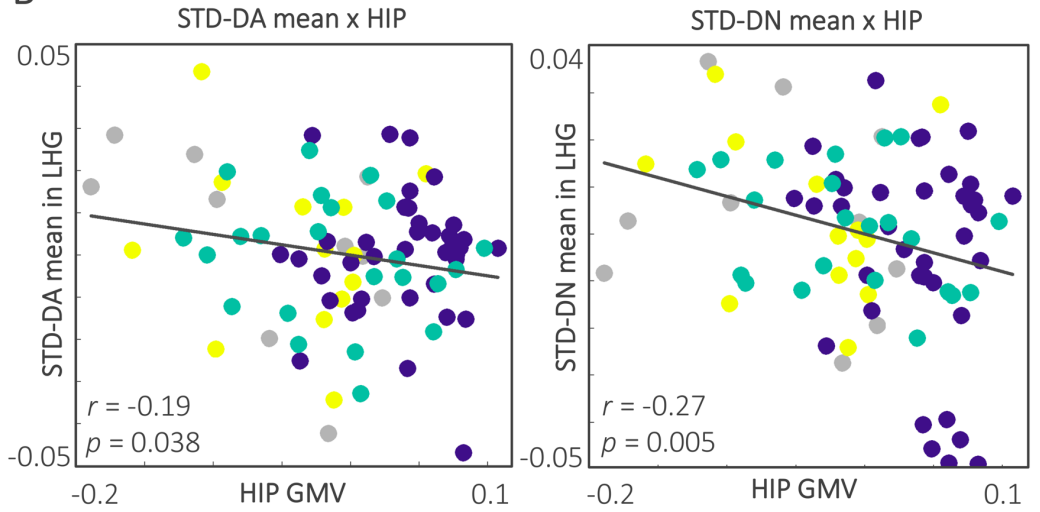

Figure 4. Neurophysiological responses are related to clinical and volumetric differences between individuals. A. The t-map displays the general linear model results across predictors and associative and novelty deviant mean responses for each region of interest (ROI). The white squares indicate significant effects. Note that the effects are stronger for the LHG across the ROls, and for the novelty deviant compared to the associative deviant. B. The scatterplots display the negative relationship between the associative and novelty deviant means in the LHG with the hippocampal GMV across the sample. ACER: Addenbrooke's Cognitive Examination Revised; ACER mem: ACER memory subscale; ENT: Entorhinal grey matter volume; GMV: Grey matter volume; HIP:

Hippocampal grey matter volume; LHG: L Heschl's gyrus; LIFG: L inferior frontal gyrus; LSTG: L superior temporal gyrus; RHG: R Heschl's gyrus; RSTG: R superior temporal gyrus; RIFG: R inferior temporal gyrus; YOE: Years of education. 


\section{Tables}

Table 1. Demographic and cognitive screening results for the four study populations.

\begin{tabular}{lllll}
\hline & Controls & Cognitive frail & $\mathrm{MCl}$ & Alzheimer \\
\hline Group size (Female) & $38(17 \mathrm{~F})$ & $26(14 \mathrm{~F})$ & $15(4 \mathrm{~F})$ & $11(6 \mathrm{~F})$ \\
Age (years) & $72.19 \pm 8.88$ & $79.98 \pm 9.50$ & $75.54 \pm 7.60$ & $74.53 \pm 11.17$ \\
Education (years) & $14.97 \pm 3.86$ & $11.07 \pm 2.88$ & $16.68 \pm 4.99$ & $11.55 \pm 3.54$ \\
Hearing left $(\mathrm{dB})$ & $51.92 \pm 13.32$ & $44.15 \pm 16.06$ & $52.88 \pm 14.77$ & $54.80 \pm 10.01$ \\
Hearing right $(\mathrm{dB})$ & $53.55 \pm 12.44$ & $43.38 \pm 16.35$ & $57.63 \pm 9.40$ & $50.00 \pm 12.01$ \\
MMSE $(/ 30)$ & $28.34 \pm 1.47$ & $26.07 \pm 2.28$ & $26.37 \pm 2.73$ & $23.20 \pm 2.78$ \\
ACE-R $(/ 100)$ & $93.71 \pm 3.04$ & $80.92 \pm 6.01$ & $83.68 \pm 8.41$ & $68.6 \pm 8.11$ \\
ACE-R memory $(/ 26)$ & $23.63 \pm 1.94$ & $18.38 \pm 3.69$ & $16.81 \pm 6.09$ & $10.7 \pm 3.43$ \\
Training test $(/ 100)$ & $65.78 \pm 23.55$ & $50.96 \pm 20.59$ & $44.16 \pm 24.02$ & $37.50 \pm 27.95$ \\
\hline
\end{tabular}

MCl: Symptomatic mild cognitive impairment after secondary/tertiary memory clinic assessment; $A C E-R$ : Revised Addenbrooke's Cognitive Examination; MMSE: Mini Mental State Examination. See Figure 2B for comparisons between groups.

Table 2. Voxel based morphometry of volume differences between groups. Columns in the table indicate the peak cluster, coordinates of the peak in $\mathrm{mm}$, the extent of the cluster, the cluster mass and corrected $\mathrm{p}$-value for the cluster respectively.

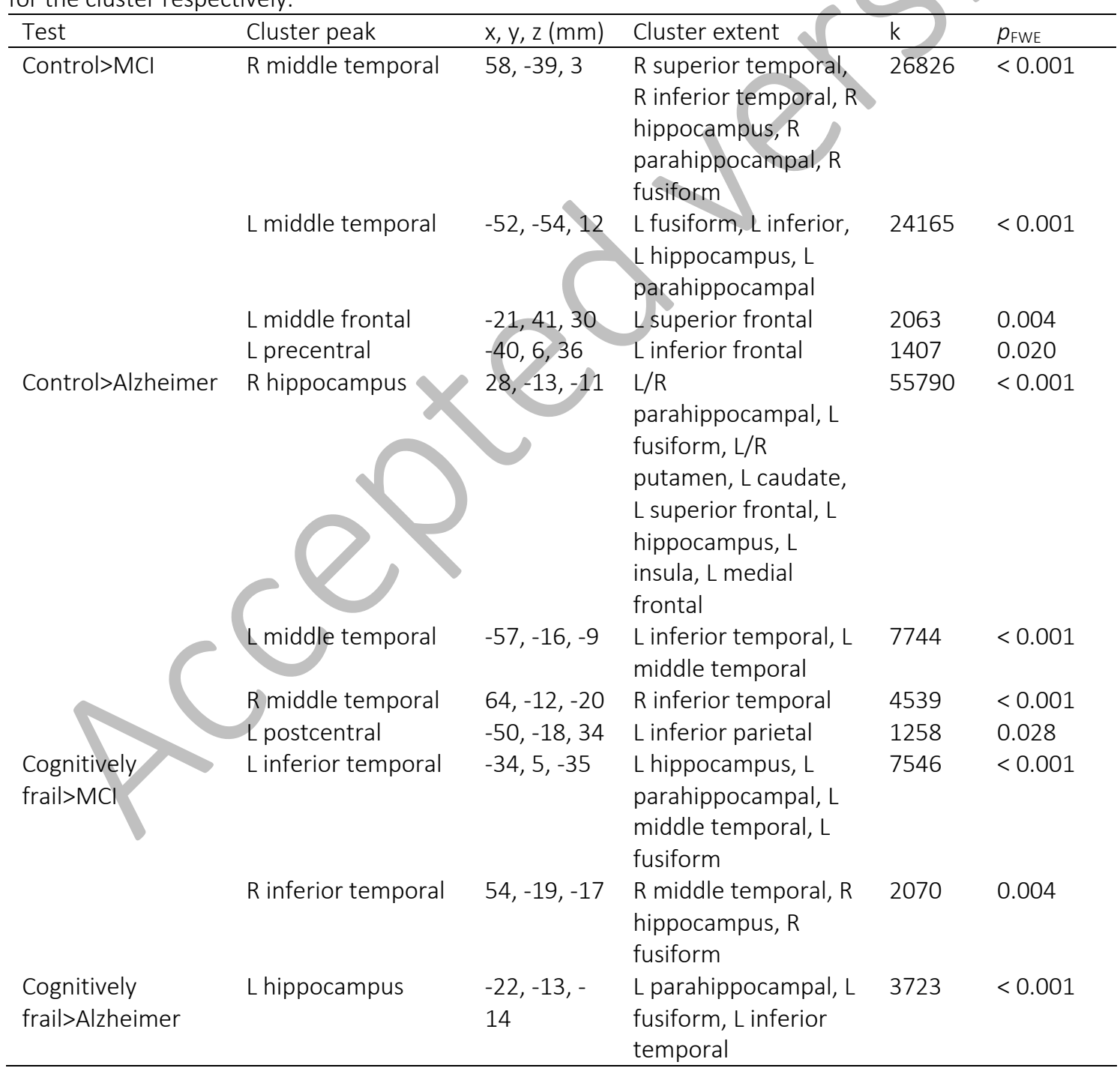

k: Cluster mass; $p_{F W E}$ : $p$-value corrected for family-wise error rate. $\mathrm{MCl}=$ symptomatic mild cognitive impairment after secondary/tertiary memory clinic assessment 
Table 3. Regional differences in the response to novelty deviants (DN) and associative deviants (DA) compared to standard trials.

\begin{tabular}{llllll}
\hline Contrast & ROI & Group & $k$ & $p_{\text {cor }}$ & Time $(\mathrm{ms})$ \\
\hline STD-DN & LIFG & Control & -1478.03 & $<0.001$ & $31-500$ \\
& & Frail & -938.79 & $<0.001$ & $175-500$ \\
& RIFG & Control & -1631.81 & $<0.001$ & $1-500$ \\
& LSTG & Control & -715.93 & 0.002 & $255-500$ \\
& & Frail & -449.99 & 0.021 & $331-500$ \\
& \multirow{2}{*}{ RSTG } & Control & -1068.44 & 0.003 & $172-500$ \\
& & Frail & -870.95 & 0.003 & $197-500$ \\
& & Alzheimer & -279.53 & 0.041 & $391-500$ \\
& LHG & Controls & -348.23 & 0.034 & $355-500$ \\
& RHG & Controls & -464.34 & 0.021 & $305-500$ \\
& & Frail & -253.10 & 0.046 & $380-500$ \\
& & MCl & -261.62 & 0.041 & $350-470$ \\
\hline STD-DA & LIFG & Control & -480.41 & 0.023 & $254-500$ \\
& & Frail & -680.74 & 0.014 & $171-497$ \\
& & MCl & -974.42 & 0.004 & $150-500$ \\
& \multirow{2}{*}{ RIFG } & Control & -944.45 & $<0.001$ & $192-500$ \\
& & Frail & -561.03 & 0.013 & $188-458$ \\
& & Alzheimer & -929.57 & 0.003 & $162-500$ \\
\hline
\end{tabular}

DA: Associative deviant; DN: Novelty deviant; k: Cluster mass; LHG: L Heschl's gyrus; LIFG: L inferior frontal gyrus; LSTG: L superior temporal gyrus; $p_{\text {cor: }}$ Corrected p-value; RHG: R Heschl's gyrus; RIFG: R inferior frontal gyrus; ROI: Regions of interest; RSTG: $R$ superior temporal gyrus; STD: Standard. See the Extended data for the log transformed version of this analysis. (Table 3-1).

Table 3-1. Regional differences in the response to novelty deviants (DN) and associative deviants (DA) compared to standard trials. In this version of the analysis, we performed the statistical comparisons on the log transformed squared RMS time series, to ensure the normality assumption of the GLMs is met. The results of the RMS and log transformed RMS time series largely overlap. However in the latter analysis task-group interaction effects do not reach statistical significance. $k$ : Cluster mass; $p_{\text {cor: }}$ Cluster corrected $p$-value.

\begin{tabular}{|c|c|c|c|c|c|}
\hline Contrast & $\mathrm{ROI}$ & Group & k & $p_{\text {cor }}$ & Time \\
\hline \multirow[t]{17}{*}{ STD-DN } & LIFG & Control & -497.43 & 0.004 & $167-252$ \\
\hline & & & -595.04 & 0.002 & $362-500$ \\
\hline & & & $-774,96$ & 0.002 & $262-500$ \\
\hline & & Control & -204.22 & 0.027 & 23-90 \\
\hline & & & -134.42 & 0.051 & $165-222$ \\
\hline & & & -594.2 & 0.004 & $343-500$ \\
\hline & LSTG & Control & -433.04 & 0.006 & $381-500$ \\
\hline & & Frail & -177.85 & 0.045 & $352-428$ \\
\hline & & & -206.56 & 0.037 & $430-500$ \\
\hline & RSTG & Control & -275.66 & 0.022 & $175-274$ \\
\hline & & & -631.41 & 0.002 & $295-500$ \\
\hline & & Frail & -274.8 & 0.018 & $260-380$ \\
\hline & & & -391.41 & 0.004 & $382-500$ \\
\hline & & Alzheimer & -182.9 & 0.041 & $438-500$ \\
\hline & LHG & Controls & -273.65 & 0.024 & $388-500$ \\
\hline & RHG & Controls & -336.9 & 0.01 & $329-487$ \\
\hline & & Frail & -138.69 & 0.043 & $1-48$ \\
\hline \multirow[t]{5}{*}{ STD-DA } & LIFG & $\mathrm{MCl}$ & -508.9 & 0.002 & $214-411$ \\
\hline & & & -155.91 & 0.047 & $454-500$ \\
\hline & RIFG & Control & -267.28 & 0.014 & $211-315$ \\
\hline & & Frail & -346.16 & 0.018 & $305-447$ \\
\hline & & Alzheimer & -775.37 & 0.008 & $177-500$ \\
\hline
\end{tabular}

